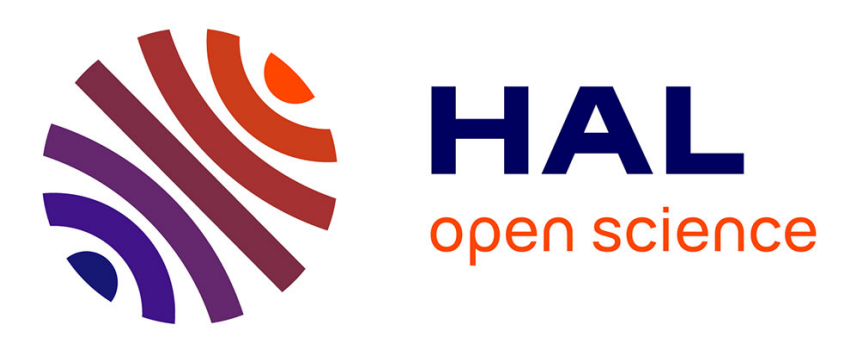

\title{
Convergence results for smooth regularizations of hybrid nonlinear optimal control problems
}

Thomas Haberkorn, Emmanuel Trélat

\section{To cite this version:}

Thomas Haberkorn, Emmanuel Trélat. Convergence results for smooth regularizations of hybrid nonlinear optimal control problems. SIAM Journal on Control and Optimization, 2011, 49 (4), pp.14981522. hal-00519458

\author{
HAL Id: hal-00519458 \\ https://hal.science/hal-00519458
}

Submitted on 20 Sep 2010

HAL is a multi-disciplinary open access archive for the deposit and dissemination of scientific research documents, whether they are published or not. The documents may come from teaching and research institutions in France or abroad, or from public or private research centers.
L'archive ouverte pluridisciplinaire HAL, est destinée au dépôt et à la diffusion de documents scientifiques de niveau recherche, publiés ou non, émanant des établissements d'enseignement et de recherche français ou étrangers, des laboratoires publics ou privés. 


\title{
CONVERGENCE RESULTS FOR SMOOTH REGULARIZATIONS OF HYBRID NONLINEAR OPTIMAL CONTROL PROBLEMS
}

\author{
T. HABERKORN AND E. TRÉLAT *
}

\begin{abstract}
We consider a class of hybrid nonlinear optimal control problems having a discontinuous dynamics ruled by a partition of the state space. For this class of problem, some hybrid versions of the usual Pontryagin Maximum Principle are known. We introduce general regularization procedures, parameterized by a small parameter, smoothing the previous hybrid problems to standard smooth optimal control problems, for which we can apply the usual Pontryagin Maximum Principle. We investigate the question of the convergence of the resulting extremals as the regularization parameter tends to zero. Under some general assumptions, we prove that smoothing regularization procedures converge, in the sense that the solution of the regularized problem (as well as its extremal lift) converges to the solution of the initial hybrid problem. To illustrate our convergence result, we apply our approach to the minimal time low-thrust coplanar orbit transfer with eclipse constraint.
\end{abstract}

Key words. Optimal control, hybrid control, regularization, shooting method, orbit transfer with eclipse.

AMS subject classifications. 49J15, 34A38, 93C65.

\section{Introduction and main results.}

1.1. Hybrid optimal control. Let $m$ and $n$ be positive integers. In what follows, $t \in \mathbb{R}$ denotes the time variable, and we consider a time dependent partition of $\mathbb{R}^{n}$,

$$
\mathbb{R}^{n}=\bigcup_{\alpha \in \mathcal{A}} \overline{X_{\alpha}(t)}
$$

where $\mathcal{A}$ is a countable set, and the subsets $X_{\alpha}(t)$ are disjoint and open with a piecewise $C^{1}$ boundary. For every $\alpha \in \mathcal{A}$, let $f_{\alpha}: \mathbb{R} \times \mathbb{R}^{n} \times \mathbb{R}^{m} \rightarrow \mathbb{R}^{n}$ and $f_{\alpha}^{0}$ : $\mathbb{R} \times \mathbb{R}^{n} \times \mathbb{R}^{m} \rightarrow \mathbb{R}$ be continuous mappings, that are $C^{1}$ with respect to their second variable. For every $t \in \mathbb{R}$, every $x \in \mathbb{R}^{n}$ and every $u \in \Omega$, define $f(t, x, u)=f_{\alpha}(t, x, u)$ and $f^{0}(t, x, u)=f_{\alpha}^{0}(t, x, u)$ whenever $x \in X_{\alpha}(t)$.

Let $\Omega$ be a measurable subset of $\mathbb{R}^{m}$. Consider the hybrid control system

$$
\dot{x}(t)=f(t, x(t), u(t)),
$$

where the control $u(\cdot)$ belongs to the class of measurable functions with values in $\Omega$. The control system (1.1) is said to be control-affine in the case where the dynamics $f$ is affine with respect to the control variable; that is, for every $\alpha \in \mathcal{A}$ there exists $m+1$ vector fields $f_{\alpha, 0}, \ldots, f_{\alpha, m}$ of class $C^{1}$ on $\mathbb{R}^{n}$ such that

$$
f_{\alpha}(t, x, u)=f_{\alpha, 0}(t, x)+\sum_{i=1}^{m} u_{i} f_{\alpha, i}(t, x),
$$

where $u=\left(u_{1}, \ldots, u_{m}\right)$. Control-affine systems are of particular interest in many applications.

Let $M_{0}$ and $M_{1}$ be two compact subsets of $\mathbb{R}^{n}$. Assume that $M_{1}$ is reachable from $M_{0}$ for the control system (1.1), in the sense that there exist a time $t_{f}>0$ and

\footnotetext{
*Université d'Orléans, Labo. MAPMO, CNRS, UMR 6628, Fédération Denis Poisson, FR 2964, Bat. Math., BP 6759, 45067 Orléans cedex 2, France. (thomas.haberkorn@univ-orleans.fr, emmanuel.trelat@univ-orleans.fr).
} 
a control $u(\cdot) \in L^{\infty}\left(\left[0, t_{f}\right], \Omega\right)$, such that the trajectory $x(\cdot)$, solution of (1.1) with $x(0) \in M_{0}$, satisfies $x\left(t_{f}\right) \in M_{1}$. Consider the optimal control problem (denoted in short (HOCP) in what follows) of steering the control system (1.1) from $M_{0}$ to $M_{1}$, and minimizing the cost function

$$
C\left(t_{f}, u(\cdot)\right)=\int_{0}^{t_{f}} f^{0}(t, x(t), u(t)) d t
$$

The final time $t_{f}$ may be fixed or not.

In [7] this kind of hybrid optimal control problem is called an optimal control problem on stratified domains, and existence of optimal control and Cauchy uniqueness results are derived using a suitable modification of usual Fillippov's arguments so as to handle the discontinuities of the dynamics and of the cost function. Note that, in that reference, the definition of stratified problem requires to define the dynamics restricted to the boundary of some domain, however we do not focus on that point here since we make an assumption of transversal crossing throughout our article (see Definition 1.1 further). Another slight difference with the framework of [7] is that our decomposition of $\mathbb{R}^{n}$ is assumed to be time dependent and our time horizon is finite, however the existence and uniqueness results of [7] are easily extended to our context.

From now on, assume that (HOCP) has a solution $(x(\cdot), u(\cdot))$ defined on $\left[0, t_{f}\right]$, with $u(\cdot) \in L^{\infty}\left(\left[0, t_{f}\right], \Omega\right)$.

For usual (smooth) optimal control problems, a well known numerical method to compute the optimal trajectory, called shooting method, consists in combining the necessary conditions derived from the Pontryagin Maximum Principle (PMP, see [27]) with a Newton method (see e.g. [35]). Let us recall how this approach has been generalized to the hybrid framework. First, the PMP has been extended to a very general hybrid context in many references (see $[6,13,16,28,32,36]$ and references therein) with different proof approaches and presentations. It is however not our aim to consider hybrid control systems in their full generality and our (HOCP) is a specific hybrid optimal control problem in the sense that the state and control spaces do not vary and the change of dynamics (1.1) is ruled by the state position and is not directly controlled. In our case, all the versions of the Hybrid Maximum Principle (denoted in short HMP) derived in the aforementioned references are equivalent and we recall hereafter a statement of the HMP applied to the optimal solution $(x(\cdot), u(\cdot))$ of (HOCP).

According to the HMP, there exist $p^{0} \leqslant 0$ and a piecewise absolutely continuous mapping $p(\cdot):\left[0, t_{f}\right] \rightarrow \mathbb{R}^{n}$ called adjoint vector, with $\left(p(\cdot), p^{0}\right) \neq(0,0)$, such that the so-called extremal $\left(x(\cdot), p(\cdot), p^{0}, u(\cdot)\right)$ is solution of

$$
\dot{x}(t)=\frac{\partial H}{\partial p}\left(t, x(t), p(t), p^{0}, u(t)\right), \quad \dot{p}(t)=-\frac{\partial H}{\partial x}\left(t, x(t), p(t), p^{0}, u(t)\right),
$$

for almost every $t \in\left[0, t_{f}\right]$, where the Hamiltonian is defined by $H\left(t, x, p, p^{0}, u\right)=$ $\langle p, f(t, x, u)\rangle+p^{0} f^{0}(t, x, u)$, and the maximization condition

$$
H\left(t, x(t), p(t), p^{0}, u(t)\right)=\max _{v \in \Omega} H\left(t, x(t), p(t), p^{0}, v\right)
$$

holds almost everywhere on $\left[0, t_{f}\right]$.

Moreover, if the final time $t_{f}$ is free, then $\max _{v \in \Omega} H\left(t_{f}, x\left(t_{f}\right), p\left(t_{f}\right), p^{0}, v\right)=0$.

In addition, for every time $t_{c}$ such that the trajectory $x(\cdot)$ passes from the domain $X_{\alpha}\left(t_{c}\right)$ to some domain $X_{\beta}\left(t_{c}\right)$, a certain jump condition holds for the adjoint vector 
at the time $t_{c}$ under some appropriate transversality condition. To make it explicit, we introduce hereafter the notion of a regular crossing time.

Definition 1.1. A regular crossing time of the trajectory $x(\cdot)$, solution of (1.1) and associated with the control $u(\cdot)$, is a time $t_{c}$ satisfying the following assumptions:

1. there exist exactly two elements $\alpha$ and $\beta$ of $\mathcal{A}$ such that the point $x\left(t_{c}\right)$ belongs to the adherence of the domains $X_{\alpha}\left(t_{c}\right)$ and $X_{\beta}\left(t_{c}\right)$;

2. there exists $\eta>0$ such that $x(t) \in X_{\alpha}(t)$ for $t \in\left(t_{c}-\eta, t_{c}\right)$ and $x(t) \in X_{\beta}(t)$ for $t \in\left(t_{c}, t_{c}+\eta\right)$;

3. the boundary between the domains $X_{\alpha}(t)$ and $X_{\beta}(t)$ can be written as $\{x \in$ $\left.\mathbb{R}^{n} \mid F(t, x)=0\right\}$ in a neighborhood of $x\left(t_{c}\right)$ and for $t$ close to $t_{c}$, with a function $F: \mathbb{R} \times \mathbb{R}^{n} \rightarrow \mathbb{R}$ of class $C^{1}$

4. the control $u(\cdot)$ is left- and right-continuous at $t_{c}$;

5. $\left\langle\partial_{x} F\left(t_{c}, x\left(t_{c}\right)\right), f_{\alpha}\left(t_{c}, x\left(t_{c}\right), u\left(t_{c}^{-}\right)\right)\right\rangle+\partial_{t} F\left(t_{c}, x\left(t_{c}\right)\right) \neq 0$;

6. $\left\langle\partial_{x} F\left(t_{c}, x\left(t_{c}\right)\right), f_{\beta}\left(t_{c}, x\left(t_{c}\right), u\left(t_{c}^{+}\right)\right)\right\rangle+\partial_{t} F\left(t_{c}, x\left(t_{c}\right)\right) \neq 0$.

The two last items of this definition represent a transversality crossing condition. Using this definition, the following jump condition holds at every regular crossing time:

$$
p\left(t_{c}^{+}\right)=p\left(t_{c}^{-}\right)+\frac{\left\langle p\left(t_{c}^{-}\right), f_{\alpha}\left(t_{c}^{-}\right)-f_{\beta}\left(t_{c}^{+}\right)\right\rangle+p^{0}\left(f_{\alpha}^{0}\left(t_{c}^{-}\right)-f_{\beta}^{0}\left(t_{c}^{+}\right)\right)}{\left\langle\partial_{x} F\left(t_{c}, x\left(t_{c}\right)\right), f_{\beta}\left(t_{c}^{+}\right)\right\rangle+\partial_{t} F\left(t_{c}, x\left(t_{c}\right)\right)} \partial_{x} F\left(t_{c}, x\left(t_{c}\right)\right) .
$$

Here, the short notation $f_{\alpha}\left(t_{c}^{-}\right)$stands for $f_{\alpha}\left(t_{c}, x\left(t_{c}\right), u\left(t_{c}^{-}\right)\right)$, and $f_{\beta}\left(t_{c}^{+}\right)$stands for $f_{\beta}\left(t_{c}, x\left(t_{c}\right), u\left(t_{c}^{+}\right)\right)$. The upperscript + (resp. - ) denotes the right (resp. left) limit.

REMARK 1.2. In the particular case where the partition of $\mathbb{R}^{n}$ into the domains $X_{\alpha}(\alpha \in \mathcal{A})$ does not depend on time, the Hamiltonian remains continuous at each boundary crossing, that is, in short, $H\left(t_{c}^{-}\right)=H\left(t_{c}^{+}\right)$.

Moreover, in that case, the two last items of Definition (1.1) mean that the left limit $\dot{x}\left(t_{c}^{-}\right)$and right limit $\dot{x}\left(t_{c}^{+}\right)$are transverse to $\partial X_{\alpha}\left(t_{c}\right)$, as illustrated on Figure 1.1. This transversality crossing assumption is crucial in our work. It discards the situations where the trajectory $x(\cdot)$ crosses a boundary between three domains or more, or hits tangentially a boundary of a domain.

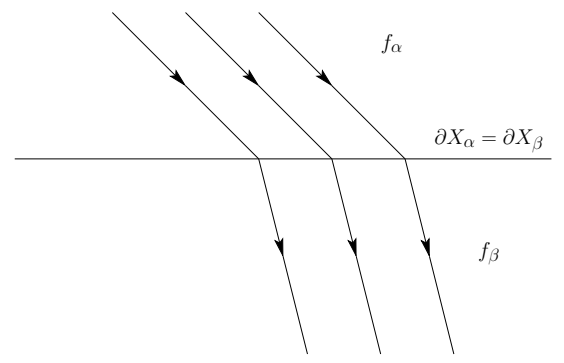

FIG. 1.1. Transversality crossing assumption

The extremal $\left(x(\cdot), p(\cdot), p^{0}, u_{1}(\cdot)\right)$ is said normal whenever $p^{0} \neq 0$, and in that case it is usual to normalize the adjoint vector so that $p^{0}=-1$; otherwise it is said abnormal.

As in the smooth case, it is then possible to derive from the HMP a (multiple) shooting method and we refer the reader e.g. to [12, 28, 32, 39] for some examples of applications. In those references, the examples are however academic and the computations can either be made by hand or the control structure (and hence the sequence 
of dynamics) can be established beforehand. Recall that the shooting methods are usually refered to as indirect methods, since they are based on the preliminary use of theoretical necessary conditions (PMP or HMP). On the opposite, direct numerical methods consist in discretizing directly the optimal control problem so as to reduce it, after discretization, to some finite dimensional nonlinear optimization problem with constraints, the dimension being as larger as the discretization is finer. Direct methods can be developed as well for solving (HOCP) by approximating it by a nonlinear programming problem (see [31] for a survey), however it is well known that such an approach cannot yield in general the degree of accuracy provided by an indirect (shooting) method. This remark is particularly relevant for problems stemming from aeronautics, such as the problem that we present in this article. The main flaw of the indirect numerical approach is that shooting methods are known to be possibly hard to initialize because they rely on a Newton like algorithm. In the hybrid context, it will be even harder to initialize because a multiple shooting has to be used to take into account the jump conditions (1.5) of the adjoint vector due to the crossings. Moreover, (1.5) is an implicit equation, because the computation of $f_{\beta}\left(t_{c}^{+}\right)$in the right hand side requires the computation of $u\left(t_{c}^{+}\right)$, itself requiring in turn the computation of $p\left(t_{c}^{+}\right)$obtained from (1.4).

The method that we propose in this article consists in regularizing (HOCP) into a smooth optimal control problem, parameterized by $\varepsilon>0$ and denoted $(\mathbf{O C P})_{\varepsilon}$, with the idea that, on the one hand $(\mathbf{O C P})_{\varepsilon}$ can be expected to be easier to solve by a numerical shooting method than (HOCP), and on the other hand nice convergence properties can be expected as $\varepsilon$ tends to zero.

The article is structured as follows. General regularization procedures are defined in Section 1.2. They are used to smooth the hybrid problem under consideration into a family of usual smooth optimal control problems parametrized by a kind of penalization parameter $\varepsilon$, and for which the PMP can be applied. Our main results, stated in Section 1.3, assert the convergence of the solution of $(\mathbf{O C P})_{\varepsilon}$ to the solution of (HOCP) (as well as their respective extremal lifts) as $\varepsilon$ tends to zero, under appropriate assumptions whose relevance is discussed in a series of remarks. Section 2 is then devoted to the proof of the main results. Finally, we illustrate in Section 3 our convergence result with a nonacademic application: the minimal time low-thrust coplanar orbit transfer around the Earth with eclipse constraint.

1.2. Regularization procedure. We first define a concept of $C^{1}$ regularization of the extended hybrid dynamics $\left(f, f^{0}\right)$.

Definition 1.3. The family $\left(f^{\varepsilon}, f^{0 \varepsilon}\right)_{\varepsilon>0}$ is called a $C^{1}$ regularization of the extended hybrid dynamics $\left(f, f^{0}\right)$ whenever the following properties are satisfied:

- for every $\varepsilon>0$, the mappings $f^{\varepsilon}: \mathbb{R} \times \mathbb{R}^{n} \times \mathbb{R}^{m} \rightarrow \mathbb{R}^{n}$ and $f^{0 \varepsilon}: \mathbb{R} \times \mathbb{R}^{n} \times$ $\mathbb{R}^{m} \rightarrow \mathbb{R}$ are $C^{1}$

- for every $t \in \mathbb{R}$, every $\alpha \in \mathcal{A}$, every $x \in X_{\alpha}(t)$, and every $u \in \Omega$, there holds

$$
\begin{aligned}
& f^{\varepsilon}(t, x, u) \underset{\varepsilon \rightarrow 0}{\longrightarrow} f_{\alpha}(t, x, u), \quad \frac{\partial f^{\varepsilon}}{\partial x}(t, x, u) \underset{\varepsilon \rightarrow 0}{\longrightarrow} \frac{\partial f_{\alpha}}{\partial x}(t, x, u), \\
& f^{0 \varepsilon}(t, x, u) \underset{\varepsilon \rightarrow 0}{\longrightarrow} f_{\alpha}^{0}(t, x, u), \quad \frac{\partial f^{0 \varepsilon}}{\partial x}(t, x, u) \underset{\varepsilon \rightarrow 0}{\longrightarrow} \frac{\partial f_{\alpha}^{0}}{\partial x}(t, x, u) .
\end{aligned}
$$

Let us provide an example of $C^{1}$ regularization.

EXAMPLE 1.4. Examples of a regularization inside a domain $X_{\alpha}(\cdot)$ are classical; for instance consider a convolution process. Let now $\bar{t} \in \mathbb{R}$ and $\bar{x}$ be a point belonging to the boundary of exactly two domains $X_{\alpha}(\bar{t})$ and $X_{\beta}(\bar{t})$, for some $\alpha, \beta \in \mathcal{A}$. Let $V$ 
be a neighborhood of $\bar{x}$ in $\mathbb{R}^{n}, d(\cdot, \cdot)$ be a distance in $\mathbb{R}^{n}$, and $b^{\varepsilon}: \mathbb{R} \times V \rightarrow[0,1]$ be a $C^{1}$ function such that

$$
b^{\varepsilon}(t, x)=\left\{\begin{array}{lll}
1 & \text { if } & x \in X_{\alpha}(t) \text { and }\left|t-t_{c}\right|+d(x, \Sigma) \geqslant \varepsilon \\
0 & \text { if } & x \in X_{\beta}(t) \text { and }\left|t-t_{c}\right|+d(x, \Sigma) \geqslant \varepsilon .
\end{array}\right.
$$

In other words, $b^{\varepsilon}$ is a kind of Heaviside function. For every $t \in \mathbb{R}$, every $x \in V$ and every $u \in \Omega$, define

$$
\begin{aligned}
f^{\varepsilon}(t, x, u) & =b^{\varepsilon}(t, x) f_{\alpha}(t, x, u)+\left(1-b^{\varepsilon}(t, x)\right) f_{\beta}(t, x, u), \\
f^{0 \varepsilon}(t, x, u) & =b^{\varepsilon}(t, x) f_{\alpha}^{0}(t, x, u)+\left(1-b^{\varepsilon}(t, x)\right) f_{\beta}^{0}(t, x, u) .
\end{aligned}
$$

This yields a local regularization on $V$. It is then easy to define a similar regularization for points belonging to the boundary of three domains or more. Then, to make it global it suffices to use for instance a partition of unit.

Let $\left(f^{\varepsilon}, f^{0 \varepsilon}\right)_{\varepsilon>0}$ be a $C^{1}$ regularization of the extended hybrid dynamics $\left(f, f^{0}\right)$. Fix $R>0$ sufficiently large. Consider the optimal control problem (denoted in short $(\mathbf{O C P})_{\varepsilon}$ in what follows) of steering the control system

$$
\dot{x}^{\varepsilon}(t)=f^{\varepsilon}\left(t, x^{\varepsilon}(t), u^{\varepsilon}(t)\right)
$$

from $M_{0}$ to $M_{1}$, with controls $u^{\varepsilon}(\cdot) \in L^{\infty}\left(\left[0, t_{f}^{\varepsilon}\right], \Omega\right)$, under the additional compact state constraint

$$
\max _{t \in\left[0, t_{f}^{\varepsilon}\right]}\left\|x^{\varepsilon}(t)\right\|_{\mathbb{R}^{n}} \leqslant R
$$

and minimizing the cost function

$$
C^{\varepsilon}\left(t_{f}^{\varepsilon}, u^{\varepsilon}(\cdot)\right)=\int_{0}^{t_{f}^{\varepsilon}} f^{0 \varepsilon}\left(t, x^{\varepsilon}(t), u^{\varepsilon}(t)\right) d t .
$$

If the final time $t_{f}$ of (HOCP) is fixed, then we set $t_{f}^{\varepsilon}=t_{f}$. If the final time $t_{f}$ of $(\mathbf{H O C P})$ is free, then the final time $t_{f}^{\varepsilon}$ of $(\mathbf{O C P})_{\varepsilon}$ is free as well; however for the problem $(\mathbf{O C P})_{\varepsilon}$ to be well defined we have to bound $t_{f}^{\varepsilon}$, and for instance we impose that $0 \leqslant t_{f}^{\varepsilon} \leqslant t_{f}+10$.

The additional constraint (1.8) is necessary to derive an existence result for $(\mathbf{O C P})_{\varepsilon}$. Adding such a constraint is a classical fact in any penalization procedure. If $R$ is chosen large enough, then, under the assumptions of our main result below, the constraint (1.8) is actually not active and hence $R$ plays no further role. In particular it does not affect the numerical process resulting from our main result (see also Remark 1.9 further).

For this regularized optimal control problem $(\mathbf{O C P})_{\varepsilon}$, anticipating the fact that the constraint (1.8) will (a posteriori) not be active, the usual PMP implies that every optimal solution $\left(x^{\varepsilon}(\cdot), u^{\varepsilon}(\cdot)\right)$ defined on $\left[0, t_{f}^{\varepsilon}\right]$ is the projection of an extremal $\left(x^{\varepsilon}(\cdot), p^{\varepsilon}(\cdot), p^{0 \varepsilon}, u^{\varepsilon}(\cdot)\right)$ solution of

$$
\dot{x}^{\varepsilon}(t)=\frac{\partial H^{\varepsilon}}{\partial p}\left(t, x^{\varepsilon}(t), p^{\varepsilon}(t), p^{0 \varepsilon}, u^{\varepsilon}(t)\right), \quad \dot{p}^{\varepsilon}(t)=-\frac{\partial H^{\varepsilon}}{\partial x}\left(t, x^{\varepsilon}(t), p^{\varepsilon}(t), p^{0 \varepsilon}, u^{\varepsilon}(t)\right),
$$

for almost every $t \in\left[0, t_{f}^{\varepsilon}\right]$, where $H^{\varepsilon}\left(t, x, p, p^{0}, u\right)=\left\langle p, f^{\varepsilon}(t, x, u)\right\rangle+p^{0} f^{0 \varepsilon}(t, x, u)$ is the Hamiltonian, $p^{0 \varepsilon}$ is a nonpositive real number, and

$$
H^{\varepsilon}\left(t, x^{\varepsilon}(t), p^{\varepsilon}(t), p^{0 \varepsilon}, u^{\varepsilon}(t)\right)=\max _{v \in \Omega} H^{\varepsilon}\left(t, x^{\varepsilon}(t), p^{\varepsilon}(t), p^{0 \varepsilon}, v\right)
$$


almost everywhere on $\left[0, t_{f}^{\varepsilon}\right]$. Moreover, if the final time is free then the maximized Hamiltonian is equal to 0 at $t_{f}^{\varepsilon}$.

Convergence properties of the solutions of $(\mathbf{O C P})_{\varepsilon}$ towards solutions of (HOCP) can be expected under appropriate assumptions. In particular, the convergence of the associated adjoint vectors, although quite difficult to prove, can be expected. This is the object of the next section, containing the main results of that paper.

1.3. Main results. Let $\left(f^{\varepsilon}, f^{0 \varepsilon}\right)_{\varepsilon>0}$ be a $C^{1}$ regularization of the extended hybrid dynamics $\left(f, f^{0}\right)$ of (HOCP). We make the following assumptions:

$\left(H_{1}\right) \Omega$ is a compact and convex subset of $\mathbb{R}^{m}$;

$\left(H_{2}\right)$ the problem (HOCP) has a unique solution $(x(\cdot), u(\cdot))$ defined on $\left[0, t_{f}\right]$;

$\left(H_{3}\right)$ the optimal trajectory $x(\cdot)$ has a unique extremal lift (up to a multiplicative scalar) on every subinterval of $\left[0, t_{f}\right]$, which is moreover normal, denoted $(x(\cdot), p(\cdot),-1, u(\cdot))$, solution of the Hybrid Maximum Principle;

$\left(H_{4}\right)$ every time $t$ such that $x(t)$ belongs to the boundary of some domain of the partition of the state space is a regular crossing time.

$\left(H_{5}\right)$ the sets

$$
\begin{aligned}
& \left\{\left(f_{\alpha}(t, x, u), f_{\alpha}^{0}(t, x, u)\right) \mid u \in \Omega\right\}, \\
& \left\{\left(f^{\varepsilon}(t, x, u), f^{0 \varepsilon}(t, x, u)\right) \mid u \in \Omega\right\}, \\
& \left\{\left(\frac{\partial f_{\alpha}}{\partial x}(t, x, u), \frac{\partial f_{\alpha}^{0}}{\partial x}(t, x, u)\right) \mid u \in \Omega\right\}, \\
& \left\{\left(\frac{\partial f^{\varepsilon}}{\partial x}(t, x, u), \frac{\partial f^{0 \varepsilon}}{\partial x}(t, x, u)\right) \mid u \in \Omega\right\},
\end{aligned}
$$

are convex, for every $t \in \mathbb{R}$, every $x \in \mathbb{R}^{n}$, every $\alpha \in \mathcal{A}$, and every $\varepsilon>0$;

$\left(H_{6}\right)$ the $C^{1}$ regularization is such that every optimal control $u^{\varepsilon}(\cdot)$ of $(\mathbf{O C P})_{\varepsilon}$ is continuous, for every $\varepsilon>0$.

THEOREM 1.5. Under the above the assumptions, there exists $\varepsilon_{0}>0$ such that, for every $\varepsilon \in\left(0, \varepsilon_{0}\right)$, the problem $(\boldsymbol{O C P P})_{\varepsilon}$ has at least one solution $\left(x^{\varepsilon}(\cdot), u^{\varepsilon}(\cdot)\right)$ defined on $\left[0, t_{f}^{\varepsilon}\right]$, every extremal lift of which is normal. Let $\left(x^{\varepsilon}(\cdot), p^{\varepsilon}(\cdot),-1, u^{\varepsilon}(\cdot)\right)$ be such a normal extremal lift. Then, as $\varepsilon$ tends to 0 ,

- $t_{f}^{\varepsilon}$ converges to $t_{f}$;

- $x^{\varepsilon}(\cdot)$ converges uniformly ${ }^{1}$ to $x(\cdot)$;

- $\dot{x}^{\varepsilon}(\cdot)$ converges to $\dot{x}(\cdot)$ in $L^{\infty}$ for the weak star topology ${ }^{2}$;

- $p^{\varepsilon}(\cdot)$ converges uniformly to $p(\cdot)$ on every closed subinterval of $\left[0, t_{f}\right]$ that does not contain any crossing time.

REMARK 1.6. Note that we are not able to derive any convergence property of the controls $u^{\varepsilon}(\cdot)$ without any further assumption. For example if we assume in addition, firstly, that for every $\varepsilon>0$, the maximization condition (1.11) can be made explicit so that $u^{\varepsilon}(t)=\varphi^{\varepsilon}\left(t, x^{\varepsilon}(t), p^{\varepsilon}(t)\right)$ for almost every $t \in\left[0, t_{f}^{\varepsilon}\right]$, secondly, that the maximization condition (1.4) can be made explicit so that $u(t)=\varphi(t, x(t), p(t))$ for almost every $t \in\left[0, t_{f}\right]$, and thirdly, that the (measurable) functions $\varphi^{\varepsilon}(\cdot, \cdot, \cdot)$ and $\varphi(\cdot, \cdot, \cdot)$ are such that $\varphi^{\varepsilon}(t, x, p)$ converges to $\varphi(t, x, p)$ as $\varepsilon$ tends to 0 for almost every $(t, x, p) \in \mathbb{R} \times \mathbb{R}^{n} \times \mathbb{R}^{n}$, then it follows immediately from the theorem that $u^{\varepsilon}(\cdot)$

\footnotetext{
${ }^{1}$ If $t_{f}^{\varepsilon}<t_{f}$, then we consider any continuous extension of $x^{\varepsilon}(\cdot)$ on $\left[0, t_{f}\right]$. The same remark holds for $\dot{x}^{\varepsilon}(\cdot), p^{\varepsilon}(\cdot)$ and $u^{\varepsilon}(\cdot)$ in the next items.

${ }^{2}$ It means that $\int_{0}^{t_{f}}\left\langle\dot{x}^{\varepsilon}(t), g(t)\right\rangle d t \rightarrow \int_{0}^{t_{f}}\langle\dot{x}(t), g(t)\rangle d t$ as $\varepsilon \rightarrow 0$, for every $g(\cdot) \in L^{1}\left(\left[0, t_{f}\right], \mathbb{R}^{n}\right)$, and where $\dot{x}^{\varepsilon}(\cdot)$ is extended continuously on $\left[0, t_{f}\right]$ if needed.
} 
converges to $u(\cdot)$ as $\varepsilon$ tends to 0 for the strong topology of $L^{1}$. This is the case e.g. when considering the minimal time problem or a quadratic criterion for control-affine systems.

Theorem 1.5 applies in particular to control-affine systems; notice that, in this case, $f_{\alpha}(t, x, \cdot)$ and $\frac{\partial f_{\alpha}}{\partial x}(t, x, \cdot)$ are convex in $u$. However we still make Assumption $\left(H_{5}\right)$ and consider control-affine systems with a general nonlinear cost. For such systems, we have the following additional result.

THEOREM 1.7. In the case of a control-affine system, under Assumptions $\left(H_{1}\right)$, $\left(H_{2}\right),\left(H_{3}\right),\left(H_{4}\right),\left(H_{5}\right)$, and the following one (instead of $\left.\left(H_{6}\right)\right)$ :

$\left(H_{6}^{\prime}\right)$ for almost every $t \in\left[0, t_{f}\right], u(t)$ is an extremal point of $\Omega$;

the conclusion of Theorem 1.5 holds and, in addition, $u^{\varepsilon}(\cdot)$ converges to $u(\cdot)$ for the strong topology of $L^{1}$.

REMARK 1.8. As mentioned previously, if the final time $t_{f}$ of (HOCP) is fixed, then $t_{f}^{\varepsilon}=t_{f}$, for every $\varepsilon>0$.

REMARK 1.9. As explained in the previous paragraph, we stress on the fact that the real number $R$ is necessary to derive an existence result for $(\mathbf{O C P})_{\varepsilon}$, but plays no role in the further analysis and in the numerical process. Since it is assumed that the solution of $(\boldsymbol{H O C P})$ is unique, it is possible to choose, for instance, $R=$ $2 \max _{t \in\left[0, t_{f}\right]}\|x(t)\|_{\mathbb{R}^{n}}$.

REMARK 1.10. The assumptions $\left(\mathrm{H}_{2}\right)$ and $\left(\mathrm{H}_{3}\right)$ on the uniqueness of the solution of $(\boldsymbol{H O C P})$ and on the uniqueness of its extremal lift are related to the differentiability properties of the value function (see for instance[3, 11], and see [9, 29, 30, 34] for results on the size of the set where the value function is differentiable).

These assumptions can be weakened as follows. If we replace Assumptions $\left(\mathrm{H}_{2}\right)$ and $\left(\mathrm{H}_{3}\right)$ with the assumption "every extremal lift of every solution of (HOCP) is normal", then the conclusion of Theorems 1.5 and 1.7 still holds, except that the convergence properties must be written in terms of closure points. More precisely, there exists $\varepsilon_{0}>0$ such that, for every $\varepsilon \in\left(0, \varepsilon_{0}\right)$, the problem $(\boldsymbol{O C P})_{\varepsilon}$ has at least one solution $\left(x^{\varepsilon}(\cdot), u^{\varepsilon}(\cdot)\right)$, every extremal lift of which is normal. For every $\varepsilon \in\left(0, \varepsilon_{0}\right)$, consider a solution $\left(x^{\varepsilon}(\cdot), u^{\varepsilon}(\cdot)\right)$ of $(\boldsymbol{O C P})_{\varepsilon}$, and let $\left(x^{\varepsilon}(\cdot), p^{\varepsilon}(\cdot),-1, u^{\varepsilon}(\cdot)\right)$ be a (normal) extremal lift. Then, every closure point of the family $\left(t_{f}^{\varepsilon}, x^{\varepsilon}(\cdot), \dot{x}^{\varepsilon}(\cdot), p^{\varepsilon}(\cdot)\right)_{0<\varepsilon<\varepsilon_{0}}$ (for the topologies considered in the statement of the theorems) can be written as $(T, \bar{x}(\cdot), \dot{\bar{x}}(\cdot), \bar{p}(\cdot))$, where $\bar{x}(\cdot)$ is solution of $(1.1)$ associated with a control $\bar{u}(\cdot) \in$ $L^{\infty}\left(\left[0, t_{f}\right], \Omega\right)$, such that $\bar{x}(0) \in M_{0}$ and $\bar{x}\left(t_{f}\right) \in M_{1}$. Moreover, $(\bar{x}(\cdot), \bar{u}(\cdot))$ is solution of $(\boldsymbol{H O C P})$ defined on $[0, T]$, having as a normal extremal lift the 4-tuple $(\bar{x}(\cdot), \bar{p}(\cdot),-1, \bar{u}(\cdot))$. The additional statement of Theorem 1.7 holds as well with an obvious adaptation in terms of closure points (see also Remark 2.8 in the proof).

REMARK 1.11. It is well known that, since the PMP is only a necessary condition for optimality, the application of a shooting method to $(\boldsymbol{O C P})_{\varepsilon}$ may only lead to an extremal solution that is not necessarily optimal. However, we have the following result (the proof of which follows from the main lines of the proof of our main results), slightly more general than Theorems 1.5 and 1.7. Assume that there is no abnormal extremal solution of the Hybrid Maximum Principle applied to (HOCP). Then, every extremal lift of every solution of $(\boldsymbol{O C P})_{\varepsilon}$ is normal, for $\varepsilon>0$ small enough. Moreover, as in Remark 1.10, every closure point of a family of such extremal solutions is a normal extremal solution of (HOCP) (for the evident topologies).

REMARK 1.12. Theorems 1.5 and 1.7 do not result from a sensitivity analysis. Indeed, we do not assume any second order sufficient condition. Usual stability and sensitivity approaches (see e.g. [14, 19, 21, 23, 25, 26] and references therein) permit 
to derive, under second order sufficient conditions assumptions, properties of Lipschitz continuous or differentiable dependence of the optimal solution and its extremal lift with respect to some parameters, for general optimal control problems. Our main results cannot be derived from such results. Our assumptions are weaker, but note that the conclusion is weaker too, since we do not prove that the solutions of $(\boldsymbol{O C P})_{\varepsilon}$ depend in a differentiable way on the parameter $\varepsilon$. Our results are rather of topological nature.

2. Proof of the main results. Our strategy of proof is the following. First, we recall the main steps of a proof of the Hybrid Maximum Principle (HMP) using needle-like variations, which are needed to derive our main result. Notice that there exists a simple proof of the HMP due to [13], that consists in reducing the problem to a usual optimal control problem with mixed initial and final conditions, and then applying the usual PMP. This simple approach is unfortunately not adapted to our problem since the regularization of the jump conditions of the HMP implies a quite difficult asymptotic study of Dirac type effects. The proof of the HMP based on needle-like variations is hence more adapted, however is more intricate than in the usual case. Indeed, when crossing the boundary of a domain, variation vectors have a jump. For the regularized problem, this turns into a difficulty of recovering, at the limit, this jump condition. In the proof of our main results, the derivation of weak convergence properties is easy and standard, using classical convexity arguments. The difficulty point is to derive a strong convergence property for the adjoint vector as well as for the control functions. To this aim, using the geometric interpretation of optimality, we derive convergence results for the Pontryagin cones, within the hybrid optimal control context. The general strategy of the proof is quite similar to the one developed in [33], however the proofs of the intermediate results are far more intricate due to the hybrid framework and the nonlinear features of the dynamics.

2.1. Preliminaries, Hybrid Maximum Principle. In this subsection, we recall the main steps of a proof of the HMP using needle-like variations.

Consider (HOCP), and introduce the instantaneous cost function $x^{0}(\cdot)$, defined on $\left[0, t_{f}\right]$ and solution of

$$
\dot{x}^{0}(t)=f^{0}(t, x(t), u(t)), x^{0}(0)=0,
$$

so that the cost $C\left(t_{f}, u(\cdot)\right)$ of the initial trajectory $x(\cdot)$ is $C\left(t_{f}, u(\cdot)\right)=x^{0}\left(t_{f}\right)$. The extended state $\tilde{x} \in \mathbb{R}^{n+1}$ is defined by $\tilde{x}=\left(x, x^{0}\right)$, and the extended dynamics by $\tilde{f}(t, \tilde{x}, u)=\left(f(t, x, u), f^{0}(t, x, u)\right)$. Consider the extended hybrid control system in $\mathbb{R}^{n+1}$,

$$
\dot{\tilde{x}}(t)=\tilde{f}(t, \tilde{x}(t), u(t)) .
$$

Let $x_{0} \in \mathbb{R}^{n}$; a control function $u(\cdot) \in L^{\infty}\left(\left[0, t_{f}\right], \mathbb{R}^{m}\right)$ is said admissible on $\left[0, t_{f}\right]$ if the trajectory $\tilde{x}(\cdot)$, solution of (2.1) associated to $u$ and such that $\tilde{x}(0)=\tilde{x}_{0}=\left(x_{0}, 0\right)$, is well defined on $\left[0, t_{f}\right]$, and the extended end-point mapping $\tilde{E}$ is then defined by $\tilde{E}\left(\tilde{x}_{0}, t_{f}, u(\cdot)\right)=\tilde{x}\left(t_{f}\right)$. The set of admissible controls on $\left[0, t_{f}\right]$ is denoted $\mathcal{U}_{\tilde{x}_{0}, t_{f}, \mathbb{R}^{m}}$, and the set of admissible controls on $\left[0, t_{f}\right]$ taking their values in $\Omega$ is denoted $\mathcal{U}_{\tilde{x}_{0}, t_{f}, \Omega}$. The set $\mathcal{U}_{\tilde{x}_{0}, t_{f}}, \mathbb{R}^{m}$, endowed with the standard topology of $L^{\infty}\left(\left[0, t_{f}\right], \mathbb{R}^{m}\right)$, is open ${ }^{3}$.

\footnotetext{
${ }^{3}$ The smoothness of the end-point mapping on the open set $\mathcal{U}_{\tilde{x}_{0}, t_{f}, \mathbb{R}^{m}}$ is a standard fact for usual control systems. It is easy to prove that this property extends to the hybrid framework under the transversality assumption $\left(H_{4}\right)$. This fact is however not used in this article.
} 
For every $t \geqslant 0$, define the extended accessible set $\tilde{A}_{\Omega}\left(\tilde{x}_{0}, t\right)$ as the image of the mapping $\tilde{E}\left(\tilde{x}_{0}, t, \cdot\right): \mathcal{U}_{\tilde{x}_{0}, t, \Omega} \rightarrow \mathbb{R}^{n+1}$, with the agreement $\tilde{A}_{\Omega}\left(\tilde{x}_{0}, 0\right)=\left\{\tilde{x}_{0}\right\}$.

Let $(x(\cdot), u(\cdot))$ be a solution of (HOCP) defined on $\left[0, t_{f}\right]$. Then the point $\tilde{x}\left(t_{f}\right)$ belongs to the boundary of the set $\tilde{A}_{\Omega}\left(\tilde{x}_{0}, t_{f}\right)$. This geometric property is at the basis of the proof of the Maximum Principle.

We next recall the concepts of needle-like variations and of Pontryagin cone, adapted to the hybrid context, which will be of crucial importance in order to prove our main result, and which also permit to derive a proof of the HMP.

In what follows, we assume that the optimal trajectory $x(\cdot)$ satisfies the transversality assumption $\left(H_{4}\right)$.

2.1.1. Needle-like variations. Let $t_{1} \in\left[0, t_{f}\right)$ and $u_{1} \in \Omega$. For $\eta>0$ such that $t_{1}+\eta \leqslant t_{f}$, the needle-like variation $\pi_{1}=\left\{t_{1}, \eta, u_{1}\right\}$ of the control $u(\cdot)$ is defined by

$$
u_{\pi_{1}}(t)= \begin{cases}u_{1} & \text { if } t \in\left[t_{1}, t_{1}+\eta\right] \\ u(t) & \text { otherwise }\end{cases}
$$

The control $u_{\pi_{1}}(\cdot)$ takes its values in $\Omega$. It is not difficult to prove that, if $\eta>0$ is small enough, then the control $u_{\pi_{1}}(\cdot)$ is admissible, i.e., the trajectory $\tilde{x}_{\pi_{1}}(\cdot)$, solution of (2.1) with the control $u_{\pi_{1}}(\cdot)$, starting from $\tilde{x}_{\pi_{1}}(0)=\tilde{x}_{0}$, is well defined on $\left[0, t_{f}\right]$. Moreover, $\tilde{x}_{\pi_{1}}(\cdot)$ converges uniformly to $\tilde{x}(\cdot)$ on $\left[0, t_{f}\right]$ whenever $\eta$ tends to 0 .

Recall that $t_{1}$ is a Lebesgue point of the function $t \mapsto \tilde{f}_{\alpha}(t, \tilde{x}(t), u(t))$ on $\left[0, t_{f}\right]$ whenever

$$
\lim _{h \rightarrow 0} \frac{1}{h} \int_{t_{1}}^{t_{1}+h} \tilde{f}_{\alpha}(t, \tilde{x}(t), u(t)) d t=\tilde{f}_{\alpha}\left(t_{1}, \tilde{x}\left(t_{1}\right), u\left(t_{1}\right)\right),
$$

and that almost every point of $\left[0, t_{f}\right]$ is a Lebesgue point.

Let $t_{1}$ be a Lebesgue point on $\left[0, t_{f}\right)$, let $\eta>0$ small enough, and $u_{\pi_{1}}(\cdot)$ be a needle-like variation of $u(\cdot)$, with $\pi_{1}=\left\{t_{1}, \eta, u_{1}\right\}$. For every $t \geqslant t_{1}$, as long as the trajectory $x(\cdot)$ remains in $X_{\alpha}(\cdot)$, the variation vector $\tilde{v}_{\pi_{1}}(\cdot)$ (not depending on $\eta$ ) is defined as the solution of the Cauchy problem

$$
\begin{aligned}
\dot{\tilde{v}}_{\pi_{1}}(t) & =\frac{\partial \tilde{f}_{\alpha}}{\partial \tilde{x}}(t, \tilde{x}(t), u(t)) \tilde{v}_{\pi_{1}}(t), \\
\tilde{v}_{\pi_{1}}\left(t_{1}\right) & =\tilde{f}_{\alpha}\left(t_{1}, \tilde{x}\left(t_{1}\right), u_{1}\right)-\tilde{f}_{\alpha}\left(t_{1}, \tilde{x}\left(t_{1}\right), u\left(t_{1}\right)\right) .
\end{aligned}
$$

Then, it is not difficult to prove that

$$
\tilde{x}_{\pi_{1}}(t)=\tilde{x}(t)+\eta \tilde{v}_{\pi_{1}}(t)+\mathrm{o}(\eta)
$$

(see e.g. [27] for details). In particular, this formula means the following. For $t_{1}$ and $u_{1}$ fixed, denote $\tilde{x}_{\alpha}(t, \eta)=\tilde{x}_{\pi_{1}}(t)$; then $\eta \mapsto \tilde{x}_{\alpha}(\cdot, \eta)$ is differentiable at 0 , and

$$
\frac{\partial \tilde{x}_{\alpha}}{\partial \eta}(t, 0)=\tilde{v}_{\pi_{1}}(t)
$$

Let us now explain how this definition of variation vector must be adapted in order to take into account the change of dynamics whenever $x(\cdot)$ leaves the domain $X_{\alpha}(\cdot)$. For $t_{1}$ and $u_{1}$ fixed, let $t(\eta)$ denote the first time at which $x_{\alpha}(t(\eta), \eta) \in \partial X_{\alpha}(t(\eta))$. Recall that, by Assumption $\left(H_{4}\right), t_{c}=t(0)$ is a regular crossing time. Assume that 
$x(\cdot)$ passes from $X_{\alpha}(\cdot)$ into $X_{\beta}(\cdot)$. In a neighborhood of $x\left(t_{c}\right)$, the boundary $\partial X_{\alpha}\left(t_{c}\right)$ can be written as $\left\{x \mid F\left(t_{c}, x\right)=0\right\}$, where $F$ is a $C^{1}$ function on $\mathbb{R}^{n+1}$. The function $(t, \eta) \mapsto x_{\alpha}(t, \eta)$ can be extended for $t \geqslant t(\eta)$, prolongating the trajectory $x_{\alpha}(\cdot)$ in the domain $X_{\beta}(\cdot)$ with the dynamics $f_{\alpha}$ (hence, $x_{\alpha}(\cdot)$ differs from the true trajectory for $t \geqslant t(\eta))$. With these notations, the crossing time $t(\eta)$ is characterized by the equality $F\left(t(\eta), x_{\alpha}(t(\eta), \eta)\right)=0$, which holds for every $\eta \geqslant 0$ small enough. Using the transversality crossing assumption $\left(H_{4}\right)$, we infer that the function $\eta \mapsto t(\eta)$ is derivable at $\eta=0$, and

$$
\left\langle\partial_{x} F\left(t_{c}, x\left(t_{c}\right)\right), t^{\prime}(0) \dot{x}\left(t_{c}^{-}\right)+\frac{\partial x_{\alpha}}{\partial \eta}\left(t_{c}, 0\right)\right\rangle+t^{\prime}(0) \partial_{t} F\left(t_{c}, x\left(t_{c}\right)\right)=0,
$$

where the upperscript - stands for the left limit, and hence

$$
t^{\prime}(0)=-\frac{\left\langle\left(\begin{array}{c}
\partial_{x} F\left(t_{c}, x\left(t_{c}\right)\right) \\
0
\end{array}\right), \tilde{v}_{\pi_{1}}\left(t_{c}^{-}\right)\right\rangle}{\left\langle\partial_{x} F\left(t_{c}, x\left(t_{c}\right)\right), f_{\alpha}\left(t_{c}^{-}, x\left(t_{c}\right), u\left(t_{c}^{-}\right)\right)\right\rangle+\partial_{t} F\left(t_{c}, x\left(t_{c}\right)\right)} .
$$

Note again that the assumption $\left(H_{4}\right)$ implies that the denominator of the above expression does not vanish.

We seek an extension of the definition of the variation vector, based on (2.4) so as to keep the validity of the expansion (2.3). Let us express the jump that is generated by the crossing. To this aim, we use the usual transport property of differential equations for the end-point mapping. Let $\delta>0$. One has $\tilde{x}_{\pi_{1}}(t(\eta)+\delta)=$ $\tilde{E}\left(x_{\pi_{1}}(t(\eta)-\delta), 2 \delta, u_{\pi_{1}}(\cdot)_{\mid[t(\eta)-\delta, t(\eta)+\delta]}\right)$, and

$$
\tilde{x}_{\pi_{1}}(t(\eta)+\delta)=\tilde{E}\left(\tilde{E}\left(\tilde{x}_{\pi_{1}}(t(\eta)-\delta), \delta, u_{\pi_{1}}(\cdot)_{\mid[t(\eta)-\delta, t(\eta)]}\right), \delta, u_{\pi_{1}}(\cdot)_{\mid[t(\eta), t(\eta)+\delta]}\right),
$$

for every $\eta>0$ small enough. On every piece, the end-point mapping is differentiable, and hence, since $\eta \mapsto t(\eta)$ is derivable, we can derivate the above equality with respect to $\eta$, and take $\eta=0$. Since one has in mind of keeping the formula (2.3), this yields

$$
\begin{aligned}
\tilde{v}_{\pi_{1}}\left(t_{c}+\delta\right)+t^{\prime}(0) & \dot{\tilde{x}}\left(t_{c}+\delta\right)=\frac{\partial \tilde{E}}{\partial x}\left(\tilde{E}\left(\tilde{x}\left(t_{c}-\delta\right), \delta, u(\cdot)_{\mid\left[t_{c}-\delta, t_{c}\right]}\right), \delta, u(\cdot)_{\mid\left[t_{c}, t_{c}+\delta\right]}\right) \\
& \circ \frac{\partial \tilde{E}}{\partial x}\left(\tilde{x}\left(t_{c}-\delta\right), \delta, u(\cdot)_{\mid\left[t_{c}-\delta, t_{c}\right]}\right) \cdot\left(t^{\prime}(0) \dot{\tilde{x}}\left(t_{c}-\delta\right)+v_{\pi_{1}}\left(t_{c}-\delta\right)\right) .
\end{aligned}
$$

Then, letting $\delta$ tend to 0 leads to $\tilde{v}_{\pi_{1}}\left(t_{c}^{+}\right)=\tilde{v}_{\pi_{1}}\left(t_{c}^{-}\right)+t^{\prime}(0)\left(\dot{\tilde{x}}\left(t_{c}^{-}\right)-\dot{\tilde{x}}\left(t_{c}^{+}\right)\right)$, that is,

$$
\tilde{v}_{\pi_{1}}\left(t_{c}^{+}\right)-\tilde{v}_{\pi_{1}}\left(t_{c}^{-}\right)=-t^{\prime}(0)\left(\tilde{f}_{\beta}\left(t_{c}^{+}, \tilde{x}\left(t_{c}\right), u\left(t_{c}^{+}\right)\right)-\tilde{f}_{\alpha}\left(t_{c}^{-}, \tilde{x}\left(t_{c}\right), u\left(t_{c}^{-}\right)\right)\right),
$$

that we denote, in short, using (2.5),

$$
\tilde{v}_{\pi_{1}}\left(t_{c}^{+}\right)=\tilde{v}_{\pi_{1}}\left(t_{c}^{-}\right)+\frac{\left\langle\left(\begin{array}{c}
\partial_{x} F\left(t_{c}, x\left(t_{c}\right)\right) \\
0
\end{array}\right), \tilde{v}_{\pi_{1}}\left(t_{c}^{-}\right)\right\rangle}{\left\langle\partial_{x} F\left(t_{c}, x\left(t_{c}\right)\right), f_{\alpha}\left(t_{c}^{-}\right)\right\rangle+\partial_{t} F\left(t_{c}, x\left(t_{c}\right)\right)}\left(\tilde{f}_{\beta}\left(t_{c}^{+}\right)-\tilde{f}_{\alpha}\left(t_{c}^{-}\right)\right) .
$$

We conclude that variation vectors of the hybrid control system (2.1) are defined by (2.2) as long as the trajectory remains in the domain $X_{\alpha}(\cdot)$, and satisfy the jump condition (2.6) whenever the trajectory crosses a boundary (with a regular transverse crossing). With that definition, the variation formula (2.3) still holds. 
REMARK 2.1. At the crossing time $t_{c}$, the following remarkable identity holds:

$$
\frac{\left\langle\left(\begin{array}{c}
\partial_{x} F\left(t_{c}, x\left(t_{c}\right)\right) \\
0
\end{array}\right), \tilde{v}_{\pi}\left(t_{c}^{-}\right)\right\rangle}{\left\langle\partial_{x} F\left(t_{c}, x\left(t_{c}\right)\right), f_{\alpha}\left(t_{c}^{-}\right)\right\rangle+\partial_{t} F\left(t_{c}, x\left(t_{c}\right)\right)}=\frac{\left\langle\left(\begin{array}{c}
\partial_{x} F\left(t_{c}, x\left(t_{c}\right)\right) \\
0
\end{array}\right), \tilde{v}_{\pi}\left(t_{c}^{+}\right)\right\rangle}{\left\langle\partial_{x} F\left(t_{c}, x\left(t_{c}\right)\right), f_{\beta}\left(t_{c}^{+}\right)\right\rangle+\partial_{t} F\left(t_{c}, x\left(t_{c}\right)\right)}
$$

Indeed, it follows immediately from the jump formula (2.6). It can also be proved by establishing the formula (2.5) with $t_{c}^{-}$replaced with $t_{c}^{+}$, and $\alpha$ replaced with $\beta$ (it suffices to consider $x_{\beta}$ instead of $\left.x_{\alpha}\right)$. The formula (2.7) is a kind of Snell-Descartes formula at the crossing time $t_{c}$. It will be of technical use in our proof.

Note that, for every $\gamma>0$, the variation $\left\{t_{1}, \gamma \eta, u_{1}\right\}$ generates the variation vector $\gamma \tilde{v}_{\pi_{1}}(\cdot)$. It follows that the set of variation vectors at time $t$ is a cone.

Definition 2.2. For every $t \in\left(0, t_{f}\right]$, the first Pontryagin cone $\tilde{K}(t) \subset \mathbb{R}^{n+1}$ at $\tilde{x}(t)$ for the extended system is defined as the smallest closed convex cone containing all variation vectors $\tilde{v}_{\pi_{1}}\left(t_{1}\right)$ for all Lebesgue points $t_{1}$ such that $0<t_{1}<t$. The first Pontryagin cone $K(t) \subset \mathbb{R}^{n}$ at $x(t)$ for the initial system is defined similarly, considering the initial dynamics $f$ instead of the extended dynamics $\tilde{f}$. Obviously, $K(t)$ is the projection on $\mathbb{R}^{n}$ of $\tilde{K}(t)$.

An immediate iteration leads to the following result, as in the usual case. Let $t_{1}<t_{2}<\cdots<t_{k}$ be Lebesgue points of the function $t \mapsto \tilde{f}(t, \tilde{x}(t), u(t))$ on $\left(0, t_{f}\right)$, and $u_{1}, \ldots, u_{k}$ be points of $\Omega$. Assume that all points $x\left(t_{i}\right)$ do not belong to the boundary of any domain $X_{\alpha}(\cdot)$ (note that, due to Assumption $\left(H_{4}\right)$, the set of such times is of full Lebesgue measure). Let $\eta_{1}, \ldots, \eta_{p}$ be small enough positive real numbers. Consider the variations $\pi_{i}=\left\{t_{i}, \eta_{i}, u_{i}\right\}$, and denote by $\tilde{v}_{\pi_{i}}(\cdot)$ the associated variation vectors, defined as above. Define the variation

$$
\pi=\left\{t_{1}, \ldots, t_{k}, \eta_{1}, \ldots, \eta_{k}, u_{1}, \ldots, u_{k}\right\}
$$

of the control $u(\cdot)$ on $\left[0, t_{f}\right]$ by

$$
u_{\pi}(t)=\left\{\begin{aligned}
u_{i} & \text { if } t_{i} \leqslant t \leqslant t_{i}+\eta_{i}, \quad i=1, \ldots, k \\
u(t) & \text { otherwise }
\end{aligned}\right.
$$

Let $\tilde{x}_{\pi}(\cdot)$ be the solution of $(2.1)$ associated with to the control $u_{\pi}(\cdot)$ on $\left[0, t_{f}\right]$ and such that $\tilde{x}_{\pi}(0)=\tilde{x}_{0}$. Then,

$$
\tilde{x}_{\pi}\left(t_{f}\right)=\tilde{x}\left(t_{f}\right)+\sum_{i=1}^{k} \eta_{i} \tilde{v}_{\pi_{i}}\left(t_{f}\right)+o\left(\sum_{i=1}^{k} \eta_{i}\right) .
$$

The first Pontryagin cone serves as an estimate of the accessible set $\tilde{A}_{\Omega}\left(\tilde{x}_{0}, t\right)$ in a neighborhood of $\tilde{x}(t)$.

When dealing with a free final time problem, we have to introduce time variations, and we rather consider the accessible set $\tilde{A}_{\Omega}\left(\tilde{x}_{0}\right)$ defined as the union of all $\tilde{A}_{\Omega}\left(\tilde{x}_{0}, s\right)$ over all $s \geqslant 0$. Assume first that $\tilde{x}(\cdot)$ is differentiable ${ }^{4}$ at time $t_{f}$. Let $\delta \in \mathbb{R}$ small enough; then, with the above notations,

$$
\tilde{x}_{\pi}\left(t_{f}+\delta\right)=\tilde{x}\left(t_{f}\right)+\sum_{i=1}^{k} \eta_{i} \tilde{v}_{\pi_{i}}\left(t_{f}\right)+\delta \tilde{f}\left(t_{f}, \tilde{x}\left(t_{f}\right), u\left(t_{f}\right)\right)+\mathrm{o}\left(\delta+\sum_{i=1}^{k} \eta_{i}\right) .
$$

\footnotetext{
${ }^{4}$ This holds true e.g. whenever $t_{f}$ is a Lebesgue point of the function $t \mapsto \tilde{f}(t, \tilde{x}(t), u(t))$.
} 
Define the cone $\tilde{K}_{1}\left(t_{f}\right)$ as the smallest closed convex cone containing $\tilde{K}\left(t_{f}\right)$ and the vectors $\pm \tilde{f}\left(t_{f}, \tilde{x}\left(t_{f}\right), u\left(t_{f}\right)\right)$. Similarly, the cone $K_{1}\left(t_{f}\right)$ is defined as the smallest closed convex cone containing $K\left(t_{f}\right)$ and the vectors $\pm f\left(t_{f}, x\left(t_{f}\right), u\left(t_{f}\right)\right)$.

If $\tilde{x}(\cdot)$ is not differentiable at time $t_{f}$, then the above construction is slightly modified, by replacing $\tilde{f}\left(t_{f}, \tilde{x}\left(t_{f}\right), u\left(t_{f}\right)\right)$ with any closure point of the corresponding difference quotient in an obvious way.

2.1.2. Conic implicit function theorem. We next provide a conic implicit function theorem, useful to derive a proof of the maximum principle.

Lemma 2.3. Let $C \subset \mathbb{R}^{m}$ be a convex subset of $\mathbb{R}^{m}$ with nonempty interior, of vertex 0 , and $F: C \rightarrow \mathbb{R}^{n}$ be a Lipschitzian mapping such that $F(0)=0$ and $F$ is differentiable in the sense of Gâteaux at 0 . Assume that $d F(0)$. Cone $(C)=\mathbb{R}^{n}$, where Cone $(C)$ stands for the (convex) cone generated by elements of $C$. Then 0 belongs to the interior of $F(\mathcal{V} \cap C)$, for every neighborhood $\mathcal{V}$ of 0 in $\mathbb{R}^{m}$.

This lemma is proved in [1] under slightly weaker assumptions and used to derive a proof of the usual Pontryagin Maximum Principle. The proof given in [1] relies on the Brouwer fixed point theorem. In the present article, we will need a version of that lemma with an additional continuous dependence on parameters. This new version is provided in Appendix (Section 4). It is however not possible to derive this parameter version from the Brouwer fixed point theorem (see Remark 4.4 for more explanations), and the proof that we provide relies on the usual Banach fixed point theorem. The assumptions of our lemma are however needed to be slightly stronger than in [1].

2.1.3. Lagrange multipliers and Hybrid Maximum Principle. We next restrict the end-point mapping to time and needle-like variations. Assume that the final time $t_{f}$ is free ${ }^{5}$. Let $k$ be a positive integer. Set

$$
\mathbb{R} \times \mathbb{R}_{+}^{k}=\left\{\left(\delta, \eta_{1}, \ldots, \eta_{k}\right) \in \mathbb{R}^{k+1} \mid \eta_{1} \geqslant 0, \ldots, \eta_{k} \geqslant 0\right\} .
$$

Let $t_{1}<\cdots<t_{k}$ be Lebesgue points of the function $t \mapsto \tilde{f}(t, \tilde{x}(t), u(t))$ on $\left(0, t_{f}\right)$, and $u_{1}, \ldots, u_{k}$ be points of $\Omega$. Let $\mathcal{V}$ be a small neighborhood of 0 in $\mathbb{R}^{k}$. Define the mapping $G: \mathcal{V} \cap\left(\mathbb{R} \times \mathbb{R}_{+}^{k}\right) \rightarrow \mathbb{R}^{n+1}$ by

$$
G\left(\delta, \eta_{1}, \ldots, \eta_{k}\right)=\tilde{x}_{\pi}\left(t_{f}+\delta\right)-\tilde{x}\left(t_{f}\right),
$$

where $\pi$ is the variation $\pi=\left\{t_{1}, \ldots, t_{k}, \eta_{1}, \ldots, \eta_{k}, u_{1}, \ldots, u_{k}\right\}$ and $|\delta|$ is small enough so that $t_{k}<t_{f}+\delta$. If $\mathcal{V}$ is small enough, then $G$ is well defined; moreover this mapping is clearly Lipschitzian, and $G(0)=0$. From (2.10), $G$ is Gâteaux differentiable on the conic neighborhood $\mathcal{V} \cap\left(\mathbb{R} \times \mathbb{R}_{+}^{k}\right)$ of 0 .

If the cone $\tilde{K}_{1}\left(t_{f}\right)$ would coincide with $\mathbb{R}^{n+1}$, then there would exist a real number $\delta$, an integer $k$ and variations $\pi_{i}=\left\{t_{i}, \eta_{i}, u_{i}\right\}, i=1, \ldots, k$, such that $G_{0}^{\prime}\left(\mathbb{R} \times \mathbb{R}_{+}^{k}\right)=$ $\mathbb{R}^{n+1}$, and then Lemma 2.3 would imply that the point $\tilde{x}\left(t_{f}\right)$ belongs to the interior of the accessible set $\tilde{A}_{\Omega}\left(\tilde{x}_{0}\right)$, which would contradict the optimality of $x(\cdot)$.

Therefore the convex cone $\tilde{K}_{1}\left(t_{f}\right)$ is not equal to $\mathbb{R}^{n+1}$. As a consequence, there exists $\tilde{\psi} \in \mathbb{R}^{n+1} \backslash\{0\}$ called Lagrange multiplier such that $\left\langle\tilde{\psi}, \tilde{v}\left(t_{f}\right)\right\rangle \leqslant 0$ for every variation vector $\tilde{v}\left(t_{f}\right) \in \tilde{K}\left(t_{f}\right)$ and $\left\langle\tilde{\psi}, \tilde{f}\left(t_{f}, \tilde{x}\left(t_{f}\right), u\left(t_{f}\right)\right)\right\rangle=0$ (at least whenever $\tilde{x}(\cdot)$

\footnotetext{
${ }^{5}$ The case where $t_{f}$ is fixed is simpler; in that case, it it not necessary to consider the real parameter $\delta$, and one uses the cone $\tilde{K}$ instead of $\tilde{K}_{1}$.
} 
is differentiable at time $t_{f}$; otherwise replace $\tilde{f}\left(t_{f}, \tilde{x}\left(t_{f}\right), u\left(t_{f}\right)\right)$ with any closure point of the corresponding difference quotient).

These inequalities then permit to derive (as in the usual way, see [27]) the statement of the HMP presented in the first section. The relation with the above Lagrange multiplier $\tilde{\psi}=\left(\psi, \psi^{0}\right)$ is that the adjoint vector $p$ can be constructed so that

$$
\psi=p\left(t_{f}\right) \quad \text { and } \quad p^{0}=\psi^{0} .
$$

In particular, the Lagrange multiplier $\psi$ is unique (up to a multiplicative scalar) if and only if the trajectory $x(\cdot)$ admits a unique extremal lift (up to a multiplicative scalar).

If $p^{0}<0$ the extremal is said normal, and in this case, since the Lagrange multiplier is defined up to a multiplicative scalar, it is usual to normalize it so that $p^{0}=-1$. If $p^{0}=0$ the extremal is called abnormal.

REMARK 2.4. The trajectory $x(\cdot)$ has an abnormal extremal lift $(x(\cdot), p(\cdot), 0, u(\cdot))$ on $\left[0, t_{f}\right]$ if and only if there exists a unit vector $\psi \in \mathbb{R}^{n}$ such that $\langle\psi, v\rangle \leqslant 0$ for every $v \in K\left(t_{f}\right)$ (and moreover $\max _{w \in \Omega}\left\langle\psi, f\left(t_{f}, x\left(t_{f}\right), w\right)\right\rangle=0$ whenever $t_{f}$ is free). In that case, one has $p\left(t_{f}\right)=\psi$, up to a multiplicative scalar.

The following lemma easily follows from the above considerations.

Lemma 2.5. Assume that, in the optimal control problem, the final time $t_{f}$ is free. For the optimal trajectory $x(\cdot)$, the following statements are equivalent:

- The trajectory $x(\cdot)$ has a unique extremal lift $\left(x(\cdot), p(\cdot), p^{0}, u(\cdot)\right.$ ) (up to a multiplicative scalar), which is moreover normal, i.e., $p^{0}<0$;

- $\tilde{K}_{1}\left(t_{f}\right)$ is a half-space of $\mathbb{R}^{n+1}$ and $p^{0}<0$;

- $\tilde{K}_{1}\left(t_{f}\right)$ is a half-space of $\mathbb{R}^{n+1}$ and $K_{1}\left(t_{f}\right)=\mathbb{R}^{n}$.

If the final time is fixed, then the above statement holds provided $\tilde{K}_{1}\left(t_{f}\right)$ is replaced with $\tilde{K}\left(t_{f}\right)$, and $K_{1}\left(t_{f}\right)$ is replaced with $K\left(t_{f}\right)$.

This important lemma permits to translate the assumptions of our main result into geometric considerations.

2.2. Proof of Theorems 1.5 an 1.7. From now on, assume that Assumptions $\left(H_{1}\right),\left(H_{2}\right),\left(H_{3}\right),\left(H_{4}\right),\left(H_{5}\right),\left(H_{6}\right)$ hold (with $\left(H_{6}\right)$ possibly replaced with $\left(H_{6}^{\prime}\right)$ in the case of a control-affine system). We denote the end-point mapping for the extended regularized system by $\tilde{E}\left(\varepsilon, x_{0}, t, u^{\varepsilon}(\cdot)\right)=\tilde{x}^{\varepsilon}(t)$, where $\tilde{x}^{\varepsilon}(\cdot)$ is the solution of the extended regularized system

$$
\dot{\tilde{x}}^{\varepsilon}(t)=\tilde{f}^{\varepsilon}\left(t, \tilde{x}^{\varepsilon}(t), u^{\varepsilon}(t)\right), \tilde{x}^{\varepsilon}(0)=\tilde{x}_{0}=\left(x_{0}, 0\right),
$$

where $\tilde{f}^{\varepsilon}\left(t, \tilde{x}^{\varepsilon}, u^{\varepsilon}\right)=\left(f^{\varepsilon}\left(t, x^{\varepsilon}, u^{\varepsilon}\right), f^{0 \varepsilon}\left(t, x^{\varepsilon}, u^{\varepsilon}\right)\right)$. By extension, the end-point mapping for the hybrid system corresponds to $\varepsilon=0$, that is $\tilde{E}\left(0, x_{0}, t, u(\cdot)\right)=\tilde{x}(t)$, where $\tilde{x}(\cdot)$ is the solution of $(2.1)$ associated with the control $u(\cdot)$ and such that $\tilde{x}(0)=\tilde{x}_{0}$. It will be also denoted $\tilde{E}\left(x_{0}, t, u(\cdot)\right)=\tilde{E}\left(0, x_{0}, t, u(\cdot)\right)=\tilde{x}(t)$.

In the sequel, we denote by $(x(\cdot), u(\cdot))$ the (unique) solution of (HOCP). We assume that the final time $t_{f}$ of $(\mathbf{H O C P})$ is free (the case of a fixed final time is similar, but simpler); as explained previously, we impose (for instance) $0 \leqslant t_{f}^{\varepsilon} \leqslant t_{f}+10$.

The proof of Theorems 1.5 and 1.7 follows from the succession of results below. Proposition 2.6 provides an existence result for solution $(\mathbf{O C P})_{\varepsilon}$, as well as first convergence properties. Proposition 2.10 and lemma 2.12 allow us to prove the existence of variation vectors of $(\mathbf{O C P})_{\varepsilon}$ that converge to variation vector of $(\mathbf{H O C P})$. Proposition 2.14 proves the normality of the extremal lifts of $(\mathbf{O C P})_{\varepsilon}$ and the boundness of its adjoint vectors. With all those ingredients we are then able to prove the theorems. 
Proposition 2.6. There exists $\varepsilon_{0}>0$ such that, for every $\varepsilon \in\left(0, \varepsilon_{0}\right)$, the problem $(\boldsymbol{O C P})_{\varepsilon}$ admits at least one solution $\left(x^{\varepsilon}(\cdot), u^{\varepsilon}(\cdot)\right)$ defined on $\left[0, t_{f}^{\varepsilon}\right]$. Moreover, $t_{f}^{\varepsilon}$ converges to $t_{f}, x^{\varepsilon}(\cdot)$ converges to $x(\cdot)$ uniformly on $\left[0, t_{f}\right], \tilde{f}^{\varepsilon}\left(\cdot, \tilde{x}^{\varepsilon}(\cdot), u^{\varepsilon}(\cdot)\right)$ converges to $\tilde{f}(\cdot, \tilde{x}(\cdot), u(\cdot))$ and $\frac{\partial \tilde{f}^{\varepsilon}}{\partial \tilde{x}}\left(\cdot, \tilde{x}^{\varepsilon}(\cdot), u^{\varepsilon}(\cdot)\right)$ converges to $\frac{\partial \tilde{f}}{\partial \tilde{x}}(\cdot, \tilde{x}(\cdot), u(\cdot))$ for the weak star topology of $L^{\infty}$, as $\varepsilon$ tends to 0 .

In the specific case of a control-affine system, under Assumption $\left(H_{6}^{\prime}\right)$ instead of $\left(H_{6}\right)$, the following additional convergence property holds: $u^{\varepsilon}(\cdot)$ converges to $u(\cdot)$ in $L^{1}\left(\left[0, t_{f}\right], \mathbb{R}^{m}\right)$ as $\varepsilon$ tends to 0 , for the strong topology.

REMARK 2.7. In particular, $\dot{x}^{\varepsilon}(\cdot)$ converges to $\dot{x}(\cdot)$ in $L^{\infty}$ for the weak star topology, as $\varepsilon$ tends to 0 .

Proof. Knowing that the constrained minimization problem (HOCP) has a solution, let us first prove that the problem $(\mathbf{O C P})_{\varepsilon}$ has at least one solution, for every $\varepsilon>0$ small enough. We use a similar reasoning as in Section 2.1.3. Let $k$ be a positive integer, $\varepsilon \geqslant 0$ and $t_{1}<\cdots<t_{k}$ be Lebesgue points of the function $t \mapsto f^{\varepsilon}\left(t, x^{\varepsilon}(t), u^{\varepsilon}(t)\right)$. Let $u_{1}, \ldots, u_{k}$ be points of $\Omega$ and $\mathcal{V}$ be a neighborhood of 0 in $\mathbb{R}^{k+2}$. Consider the variation $\pi=\left\{t_{1}, \ldots, t_{k}, \eta_{1}, \ldots, \eta_{k}, u_{1}, \ldots, u_{k}\right\}$ of the control $u(\cdot)$, and define the associated mapping $\Gamma: \mathcal{V} \cap\left(\mathbb{R}_{+} \times \mathbb{R} \times \mathbb{R}_{+}^{k}\right) \rightarrow \mathbb{R}^{n}$ by

$$
\Gamma\left(\varepsilon, \delta, \eta_{1}, \ldots, \eta_{k}\right)=x_{\pi}^{\varepsilon}\left(t_{f}+\delta\right)-x\left(t_{f}\right) .
$$

From Assumption $\left(H_{3}\right)$, the unique extremal lift of $x(\cdot)$ is normal, hence it follows from Lemma 2.5 that $K_{1}\left(t_{f}\right)=\mathbb{R}^{n}$. Therefore, there exist a real number $\delta$, an integer $k$ and a variation $\pi=\left\{t_{1}, \ldots, t_{k}, \eta_{1}, \ldots, \eta_{k}, u_{1}, \ldots, u_{k}\right\}$ such that the associated mapping $\Gamma$ satisfies

$$
\frac{\partial \Gamma}{\partial\left(\delta, \eta_{1}, \ldots, \eta_{k}\right)}(0) .\left(\mathbb{R} \times \mathbb{R}_{+}^{k}\right)=K_{1}\left(t_{f}\right)=\mathbb{R}^{n} .
$$

The conic implicit function theorem with parameters, Theorem 4.1 of the Appendix (Section 4), implies that there exist $\varepsilon_{0}>0$, such that for every $\varepsilon \in\left[0, \varepsilon_{0}\right)$, there exist $\delta_{\varepsilon} \in \mathbb{R}$ and a variation $\pi_{\varepsilon}=\left\{t_{1}^{\varepsilon}, \ldots, t_{k}^{\varepsilon}, \eta_{1}^{\varepsilon}, \ldots, \eta_{k}^{\varepsilon}, u_{1}^{\varepsilon}, \ldots, u_{k}^{\varepsilon}\right\}$ such that there holds $\Gamma\left(\varepsilon, \delta_{\varepsilon}, \eta_{1}^{\varepsilon}, \ldots, \eta_{k}^{\varepsilon}\right)=0$, and moreover $\left|\delta_{\varepsilon}\right|$ is small whenever $\varepsilon_{0}$ is small enough. In other words, for every $\varepsilon>0$ small enough, the subset $M_{1}$ is reachable from the subset $M_{0}$ for the regularized control system (1.7), within a time $t_{f}^{\varepsilon} \in\left[0, t_{f}+10\right]$, and with the control $u_{\pi_{\varepsilon}}(\cdot) \in L^{\infty}\left(\left[0, t_{f}^{\varepsilon}\right], \Omega\right)$.

The existence of an optimal control steering the regularized system from $M_{0}$ to $M_{1}$ is then a standard fact to derive, using the convexity assumptions $\left(H_{5}\right)$ on the extended velocities, the compactness of $M_{0}$ and $M_{1}$, and the additional compactness assumption (1.8) (see e.g. [10] for such existence results).

Let us now prove the convergence properties. Although the reasoning is also quite straightforward, we include however a proof since the result does not follow directly from standard results, due to the fact that the dynamics depends on the parameter $\varepsilon$. Let $\left(\varepsilon_{k}\right)_{k \in \mathbb{N}}$ be an arbitrary sequence of positive real numbers converging to 0 as $k$ tends to $+\infty$. Since $t_{f}^{\varepsilon_{k}} \in\left[0, t_{f}+10\right]$, the sequence $\left(t_{f}^{\varepsilon_{k}}\right)_{k \in \mathbb{N}}$ converges, up to a subsequence, to some $T \in\left[0, t_{f}+10\right]$. Since $M_{0}$ and $M_{1}$ are compact, and $x^{\varepsilon_{k}}(0) \in M_{0}$ and $x^{\varepsilon_{k}}\left(t_{f}^{\varepsilon_{k}}\right) \in M_{1}$, the sequences $\left(x^{\varepsilon_{k}}(0)\right)_{k \in \mathbb{N}}$ and $\left(x^{\varepsilon_{k}}\left(t_{f}^{\varepsilon_{k}}\right)\right)_{k \in \mathbb{N}}$ converge up to a subsequence respectively to some $\bar{x}_{0} \in M_{0}$ and $\bar{x}_{T} \in M_{1}$.

For every integer $k$ and almost every $t \in\left[0, t_{f}^{\varepsilon_{k}}\right]$, set $g_{k}(t)=f^{\varepsilon_{k}}\left(t, x^{\varepsilon_{k}}(t), u^{\varepsilon_{k}}(t)\right)$. Assumption $\left(H_{1}\right)$ and the state constraint (1.8) imply that the sequence $\left(g_{k}(\cdot)\right)_{k \in \mathbb{N}}$ is bounded in $L^{\infty}$, hence up to a subsequence it converges to some $g(\cdot) \in L^{\infty}\left([0, T], \mathbb{R}^{n}\right)$ 
for the weak star topology. For every $t \in[0, T]$, set $\bar{x}(t)=\bar{x}_{0}+\int_{0}^{t} g(s) d s$. Since $x^{\varepsilon_{k}}(t)=\bar{x}_{0}+\int_{0}^{t} g_{k}(s) d s$, it follows that $x^{\varepsilon_{k}}(\cdot)$ converges uniformly to $\bar{x}(\cdot)$, up to a subsequence. In particular, $\bar{x}(\cdot)$ satisfies the state constraint $\max _{t \in[0, T]}\|\bar{x}(t)\|_{\mathbb{R}^{n}} \leqslant R$.

Let us prove that there exists a control $\bar{u}(\cdot) \in L^{\infty}([0, T], \Omega)$ such that $\bar{x}(\cdot)$ is solution of the control system (1.1), associated with the control $\bar{u}(\cdot)$. Note that, using Assumptions $\left(H_{1}\right)$ and $\left(H_{5}\right), g_{k}(t) \in V^{\varepsilon_{k}}\left(t, x^{\varepsilon_{k}}(t)\right)$, for every integer $k$ and almost every $t \in\left[0, t_{f}^{\varepsilon_{k}}\right]$, where $V^{\varepsilon_{k}}\left(t, x^{\varepsilon_{k}}(t)\right)=\left\{f^{\varepsilon_{k}}\left(t, x^{\varepsilon_{k}}(t), u\right) \mid u \in \Omega\right\}$ is a compact and convex subset of $\mathbb{R}^{n}$. To prove the statement, let us first prove that $g(t) \in V(t, \bar{x}(t))$ for almost every $t \in[0, T]$, where $V(t, \bar{x}(t))=\{f(t, \bar{x}(t), u) \mid u \in \Omega\}$. Note that $V(t, \bar{x}(t))$ is a compact and convex subset of $\mathbb{R}^{n}$. It follows from the definition of a $C^{1}$ regularization (Definition 1.1) and from the convexity assumptions that the sequence of compact convex subsets $\left(V^{\varepsilon_{k}}\left(t, x^{\varepsilon_{k}}(t)\right)\right)_{k \in \mathbb{N}}$ converges (in the usual sense of Hausdorff) to $V(t, \bar{x}(t))$ for almost every $t$. For every $\delta \geqslant 0$, set

$$
\mathcal{V}_{\delta}=\left\{h(\cdot) \in L^{2}\left([0, T], \mathbb{R}^{n}\right) \mid h(t) \in V_{\delta}(t, \bar{x}(t)) \text { for almost every } t \in\left[0, t_{f}\right]\right\},
$$

where $V_{\delta}(t, x(t))$ is the compact convex set consisting of the points of $\mathbb{R}^{n}$ that are at a distance of $V(t, x(t))$ less than or equal to $\delta$. It is not difficult to see that, for every $\delta \geqslant 0, \mathcal{V}_{\delta}$ is a closed convex subset of $L^{2}\left([0, T], \mathbb{R}^{n}\right)$ for the strong topology, and thus as well for the weak topology. Let $\delta>0$ arbitrary. Note that $g_{k}(\cdot) \in \mathcal{V}_{\delta}$ whenever $k$ is large enough. Since the sequence $\left(g_{k}(\cdot)\right)_{k \in \mathbb{N}}$ converges up to a subsequence to $g(\cdot)$ for the weak star topology of $L^{\infty}$, it converges as well up to a subsequence to $g(\cdot)$ for the weak topology of $L^{2}$. Using the closedness of $V_{\delta}$ for this topology, we infer that $g(\cdot) \in V_{\delta}$. Since $\delta>0$ is arbitrary, it follows that $g(\cdot) \in V_{0}$, that is, for almost every $t \in[0, T]$ there exists $\bar{u}(t) \in \Omega$ such that $g(t)=f(t, \bar{x}(t), \bar{u}(t))$. The fact that the function $\bar{u}(\cdot)$ can be chosen to be measurable on $[0, T]$ follows form a standard measurable selection lemma (see e.g. [22, Lemma 3A page 161]).

Repeating all previous arguments for the extended systems (replacing $x$ with $\tilde{x}$ and $x^{\varepsilon}$ with $\left.\tilde{x}^{\varepsilon}\right)$ permits to show as well that $C^{\varepsilon_{k}}\left(t_{f}^{\varepsilon_{k}}, u^{\varepsilon_{k}}\right)$ converges to $C(T, \bar{u})$ as $k$ tends to $+\infty$. Since $\left(x^{\varepsilon_{k}}(\cdot), u^{\varepsilon_{k}}(\cdot)\right)$ is the optimal solution of $(\mathbf{O C P})_{\varepsilon}$, there holds $C^{\varepsilon_{k}}\left(t_{f}^{\varepsilon_{k}}, u^{\varepsilon_{k}}\right) \leqslant C^{\varepsilon_{k}}(\tau, v)$ for every $\tau \geqslant 0$ and every control $v \in L^{\infty}([0, \tau], \Omega)$ steering the regularized system (1.7) from $M_{0}$ to $M_{1}$. It then follows in particular that $C(T, \bar{u}) \leqslant C\left(t_{f}, u\right)$, and from the uniqueness assumption $\left(H_{2}\right)$, we infer that $T=t_{f}$, $\bar{u}(\cdot)=u(\cdot)$ almost everywhere and $\bar{x}(\cdot)=x(\cdot)$ on $\left[0, t_{f}\right]$.

Similarly, using the convexity assumption $\left(H_{5}\right)$, the previous argumentation can be developed to derive convergence properties for the sequence $\frac{\partial \tilde{f}^{\varepsilon_{k}}}{\partial \tilde{x}}\left(\cdot, \tilde{x}^{\varepsilon_{k}}(\cdot), u^{\varepsilon_{k}}(\cdot)\right)$.

Hence, at this step, we have proved that, up to a subsequence, $\left(t_{f}^{\varepsilon_{k}}\right)_{k \in \mathbb{N}}$ converges to $t_{f},\left(x^{\varepsilon_{k}}(\cdot)\right)_{k \in \mathbb{N}}$ converges to $x(\cdot)$ uniformly on $\left[0, t_{f}\right],\left(\tilde{f}^{\varepsilon_{k}}\left(\cdot, x^{\varepsilon_{k}}(\cdot), u^{\varepsilon_{k}}(\cdot)\right)\right)_{k \in \mathbb{N}}$ converges to $\tilde{f}(\cdot, x(\cdot), u(\cdot))$ and $\left(\frac{\partial \tilde{f}^{\varepsilon_{k}}}{\partial \tilde{x}}\left(\cdot, x^{\varepsilon_{k}}(\cdot), u^{\varepsilon_{k}}(\cdot)\right)\right)_{k \in \mathbb{N}}$ converges to $\frac{\partial \tilde{f}}{\partial \tilde{x}}(\cdot, x(\cdot), u(\cdot))$ in $L^{\infty}$ for the weak star topology, as $k$ tends to $+\infty$.

Let us now investigate the convergence of the sequence $\left(u^{\varepsilon_{k}}(\cdot)\right)_{k \in \mathbb{N}}$ in the specific case of a control-affine system and under Assumption $\left(H_{6}^{\prime}\right)$ instead of $\left(H_{6}\right)$. For a control-affine system, we infer from the above convergence properties that the sequence $\left(u^{\varepsilon_{k}}(\cdot)\right)_{k \in \mathbb{N}}$ converges up to a subsequence to $u(\cdot)$ for the weak star topology of $L^{\infty}$, and thus as well for the weak topology of $L^{2}$. Besides, from Assumption $\left(H_{6}^{\prime}\right)$, $u(t)$ is an extremal point of $\Omega$, for almost every $t \in\left[0, t_{f}\right]$. It then follows from [38, Corollary 1] that $u^{\varepsilon_{k}}(\cdot)$ converges strongly (up to a subsequence) to $u(\cdot)$ in $L^{1}$.

To conclude, we have shown that $\left(t_{f}, x(\cdot), \dot{x}(\cdot)\right)$ (resp. $\left(t_{f}, x(\cdot), u(\cdot)\right)$ for the specific case of a control-affine system under Assumption $\left(H_{6}^{\prime}\right)$ ) is the unique closure point (for the topologies used above) of the sequence $\left(t_{f}^{\varepsilon_{k}}, x^{\varepsilon_{k}}(\cdot), \dot{x}^{\varepsilon_{k}}(\cdot)\right)_{k \in \mathbb{N}}$ 
(resp. $\left(t_{f}^{\varepsilon_{k}}, x^{\varepsilon_{k}}(\cdot), u^{\varepsilon_{k}}(\cdot)\right)_{k \in \mathbb{N}}$ ), where $\left(\varepsilon_{k}\right)_{k \in \mathbb{N}}$ is any sequence of positive real numbers converging to 0 , and therefore the convergence holds as well for the whole family $\left(t_{f}^{\varepsilon}, x^{\varepsilon}(\cdot), \dot{x}^{\varepsilon}(\cdot)\right)_{0<\varepsilon<\varepsilon_{0}}\left(\operatorname{resp} .\left(t_{f}^{\varepsilon}, x^{\varepsilon}(\cdot), u^{\varepsilon}(\cdot)\right)_{0<\varepsilon<\varepsilon_{0}}\right)$.

REMARK 2.8. If one does not assume the uniqueness of the optimal solution of $(\boldsymbol{H O C P})$, then the previous proof implies that the following statement still holds: every closure point of the family $\left(t_{f}^{\varepsilon}, x^{\varepsilon}(\cdot), \dot{x}^{\varepsilon}(\cdot), p^{\varepsilon}(\cdot)\right)_{0<\varepsilon<\varepsilon_{0}}$ (for the topologies considered above) can be written as $(T, \bar{x}(\cdot), \dot{\bar{x}}(\cdot), \bar{p}(\cdot))$, where $\bar{x}(\cdot)$ is solution of (1.1) associated with a control $\bar{u}(\cdot) \in L^{\infty}\left(\left[0, t_{f}\right], \Omega\right)$, such that $\bar{x}(0) \in M_{0}$ and $\bar{x}\left(t_{f}\right) \in M_{1}$. Moreover, $(\bar{x}(\cdot), \bar{u}(\cdot))$ is another possible solution of $(\boldsymbol{H O C P})$ defined on $[0, T]$, having as a normal extremal lift the 4-tuple $(\bar{x}(\cdot), \bar{p}(\cdot),-1, \bar{u}(\cdot))$. Furthermore, for a controlaffine system under Assumption $\left(H_{6}^{\prime}\right)$, the family $\left(u^{\varepsilon}(\cdot)\right)_{0<\varepsilon<\varepsilon_{0}}$ has the closure point $\bar{u}(\cdot) \in L^{1}([0, T], \Omega)$ for the strong topology.

In other words, every closure point of a family of solutions of $(\boldsymbol{O C P})_{\varepsilon}$ is a solution of (HOCP).

REMARK 2.9. The solution of $(\boldsymbol{O C P})_{\varepsilon}$ is not necessarily unique. However all results that follow do not depend on the specific choice of a solution.

In the sequel, let $\left(x^{\varepsilon}(\cdot), u^{\varepsilon}(\cdot)\right)$ be a solution of $(\mathbf{O C P})_{\varepsilon}$ defined on $\left[0, t_{f}^{\varepsilon}\right]$, for every $\varepsilon \in\left(0, \varepsilon_{0}\right)$. Since $x^{\varepsilon}(\cdot)$ converges uniformly to $x(\cdot)$, if we choose $R>0$ large enough (see Remark 1.9) then the state constraint (1.8) is not active in $(\mathbf{O C P})_{\varepsilon}$, as announced in Section 1.2. It then follows from the usual Pontryagin Maximum Principle applied to that $x^{\varepsilon}(\cdot)$ is the projection of an extremal $\left(x^{\varepsilon}(\cdot), p^{\varepsilon}(\cdot), p^{0 \varepsilon}, u^{\varepsilon}(\cdot)\right)$ satisfying (1.10) and (1.11).

In order to derive convergence properties for the adjoint vector, we come back to the geometric interpretation of the proof of the PMP or HMP in terms of Pontryagin cones, as explained formerly. In what follows, we use the Pontryagin cones $K(t)$, $K_{1}(t), \tilde{K}(t), \tilde{K}_{1}(t)$ along the trajectory $x(\cdot)$ solution of (HOCP), introduced in the previous subsection. Similarly, for every $\varepsilon>0$, we denote by $K^{\varepsilon}(t), K_{1}^{\varepsilon}(t), \tilde{K}^{\varepsilon}(t)$, $\tilde{K}_{1}^{\varepsilon}(t)$ the Pontryagin cones along the trajectory $x^{\varepsilon}(\cdot)$. The following result states nice convergence properties for the Pontryagin cones.

Proposition 2.10. For every $\tilde{v} \in \tilde{K}\left(t_{f}\right)$, for every $\varepsilon>0$, there exists $\tilde{v}^{\varepsilon} \in$ $\tilde{K}^{\varepsilon}\left(t_{f}^{\varepsilon}\right)$ such that $\tilde{v}^{\varepsilon}$ converges to $\tilde{v}$ as $\varepsilon$ tends to 0 .

Proof. By construction of $\tilde{K}\left(t_{f}\right)$, it suffices to prove the lemma for a single needlelike variation. Assume that $\tilde{v}=\tilde{v}_{\pi}\left(t_{f}\right)$, where the variation vector $\tilde{v}_{\pi}(\cdot)$ is the solution on $\left[t_{1}, t_{f}\right]$ of the Cauchy problem

$$
\begin{aligned}
\dot{\tilde{v}}_{\pi}(t) & =\frac{\partial \tilde{f}_{\alpha}}{\partial \tilde{x}}(t, \tilde{x}(t), u(t)) . \tilde{v}_{\pi}(t), \\
\tilde{v}_{\pi}\left(t_{1}\right) & =\tilde{f}\left(t_{1}, \tilde{x}\left(t_{1}\right), u_{1}\right)-\tilde{f}\left(t_{1}, \tilde{x}\left(t_{1}\right), u\left(t_{1}\right)\right),
\end{aligned}
$$

as long as $x(t) \in X_{\alpha}(t)$, where $t_{1}$ is a Lebesgue point of $\left[0, t_{f}\right), u_{1} \in \Omega$, and the needle-like variation $\pi=\left\{t_{1}, \eta, u_{1}\right\}$ of the control $u(\cdot)$ is defined by

$$
u_{\pi}(t)= \begin{cases}u_{1} & \text { if } t \in\left[t_{1}, t_{1}+\eta\right] \\ u(t) & \text { otherwise }\end{cases}
$$

When $x(\cdot)$ crosses (transversally) the boundary of $X_{\alpha}(\cdot)$, the variation vector $\tilde{v}_{\pi}(\cdot)$ satisfies the jump condition (2.6).

In order to define a needle-like variation of the regularized control $u^{\varepsilon}(\cdot)$, we first need the following technical lemma. 
Lemma 2.11. For almost every $t \in\left(0, t_{f}\right)$, there exists a family $\left(t^{\varepsilon}\right)_{\varepsilon>0}$ of points of $\left[t, t_{f}\right)$ such that $t^{\varepsilon} \rightarrow t$ and $\tilde{f}^{\varepsilon}\left(t^{\varepsilon}, \tilde{x}^{\varepsilon}\left(t^{\varepsilon}\right), u^{\varepsilon}\left(t^{\varepsilon}\right)\right) \rightarrow \tilde{f}(t, \tilde{x}(t), u(t))$ as $\varepsilon \rightarrow 0$, and such that $t^{\varepsilon}$ is a Lebesgue point of the function $t \mapsto \tilde{f}^{\varepsilon}\left(t, \tilde{x}^{\varepsilon}(t), u^{\varepsilon}(t)\right)$.

Proof. [Proof of Lemma 2.11] Set $h^{\varepsilon}(t)=\tilde{f}^{\varepsilon}\left(t, \tilde{x}^{\varepsilon}(t), u^{\varepsilon}(t)\right)$ and $h(t)=\tilde{f}(t, \tilde{x}(t), u(t)$, and denote $h^{\varepsilon}(t)=\left(h_{1}^{\varepsilon}(t), \ldots, h_{k+1}^{\varepsilon}(t)\right)$ and $h(t)=\left(h_{1}(t), \ldots, h_{k+1}(t)\right)$ their coordinates in $\mathbb{R}^{n+1}$. Let us prove that, for almost every $t \in\left(0, t_{f}\right)$, for every $\beta>0$ and every $\alpha>0$ (small enough so that $t+\alpha<t_{f}$ ), there exists $\gamma>0$ such that, for every $\varepsilon \in(0, \gamma)$, there exists $t^{\varepsilon} \in[t, t+\alpha]$ such that $\left\|h^{\varepsilon}\left(t^{\varepsilon}\right)-h(t)\right\| \leqslant \beta$ (here, $\|\cdot\|$ denotes a norm in $\left.\mathbb{R}^{n+1}\right)$.

The proof goes by contradiction. Assume that there exists a measurable subset $A$ of $\left(0, t_{f}\right)$ of positive measure such that, for every $t \in A$, there exist $\beta>0$ and $\alpha>0$ such that, for every integer $k$, there exist $\varepsilon_{k} \in(0,1 / k)$ and $i \in\{1, \ldots, n+1\}$ such that, for every $s \in[t, t+\alpha]$, there holds

$$
\left|h_{i}^{\varepsilon_{k}}(s)-h_{i}(t)\right|>\beta .
$$

We distinguish between two cases, depending on whether Assumption $\left(H_{6}\right)$ or Assumption $\left(H_{6}^{\prime}\right)$ holds.

We first assume that Assumption $\left(H_{6}\right)$ holds, that is, $u^{\varepsilon}(\cdot)$ is continuous for every $\varepsilon>0$. This implies that $h^{\varepsilon}(\cdot)$ is continuous as well, for every $\varepsilon>0$. It follows from the proof of the previous proposition that the family $\left(h^{\varepsilon}(\cdot)\right)_{0<\varepsilon<\varepsilon_{0}}$ converges to $h(\cdot)$ in $L^{\infty}$ for the weak star topology, and hence its restriction to interval converges as well to the corresponding restriction of $h(\cdot)$. Since $h_{i}^{\varepsilon_{k}}(\cdot)$ is continuous, we infer from (2.14) that either $h_{i}^{\varepsilon_{k}}(s) \geqslant h_{i}(t)+\beta$ for every $s \in[t, t+\alpha]$, or $h_{i}^{\varepsilon_{k}}(s) \leqslant h_{i}(t)-\beta$ for every $s \in[t, t+\alpha]$. This inequality contradicts the weak convergence of the restriction to $[t, t+\alpha]$ of $h_{i}^{\varepsilon_{k}}(\cdot)$ towards the restriction to $[t, t+\alpha]$ of $h_{i}(\cdot)$.

In the second case, under Assumption $\left(H_{6}^{\prime}\right)$ instead of $\left(H_{6}\right)$ (and for a controlaffine system), we have proved previously that the family $\left(u^{\varepsilon}(\cdot)\right)_{0<\varepsilon<\varepsilon_{0}}$ converges to $u(\cdot)$ for the strong topology of $L^{1}$. Therefore, up to a subsequence the sequence $\left(u^{\varepsilon_{k}}(\cdot)\right)_{k \in \mathbb{N}}$ converges almost everywhere to $u(\cdot)$. We infer that the sequence $\left(h_{i}^{\varepsilon_{k}}(\cdot)\right)_{k \in \mathbb{N}}$ converges almost everywhere, up to a subsequence, to $h_{i}(\cdot)$ This raises a contradiction with (2.14). $\mathrm{c}$

From Lemma 2.11, for every $\varepsilon>0$ small enough, there exists $t_{1}^{\varepsilon} \geqslant t_{1}$ such that $t_{1}^{\varepsilon} \rightarrow t_{1}$ and $\tilde{f}^{\varepsilon}\left(t_{1}^{\varepsilon}, \tilde{x}^{\varepsilon}\left(t_{1}^{\varepsilon}\right), u^{\varepsilon}\left(t_{1}^{\varepsilon}\right)\right) \rightarrow \tilde{f}\left(t_{1}, \tilde{x}\left(t_{1}\right), u\left(t_{1}\right)\right)$ as $\varepsilon \rightarrow 0$. Consider then the needle-like variation $\pi^{\varepsilon}=\left\{t_{1}^{\varepsilon}, \eta, u_{1}\right\}$ of the control $u^{\varepsilon}(\cdot)$ defined by ${ }^{6}$

$$
u_{\pi^{\varepsilon}}^{\varepsilon}(t)= \begin{cases}u_{1} & \text { if } t \in\left[t_{1}^{\varepsilon}, t_{1}^{\varepsilon}+\eta\right] \\ u^{\varepsilon}(t) & \text { otherwise }\end{cases}
$$

and define the variation vector $\tilde{v}_{\pi^{\varepsilon}}(\cdot)$ as the solution on $\left[t_{1}^{\varepsilon}, t_{f}^{\varepsilon}\right]$ of the Cauchy problem

$$
\begin{aligned}
\dot{\tilde{v}}_{\pi^{\varepsilon}}(t) & =\frac{\partial \tilde{f}^{\varepsilon}}{\partial \tilde{x}}\left(t, \tilde{x}^{\varepsilon}(t), u^{\varepsilon}(t)\right) \cdot \tilde{v}_{\pi^{\varepsilon}}(t) \\
\tilde{v}_{\pi^{\varepsilon}}\left(t_{1}^{\varepsilon}\right) & =\tilde{f}^{\varepsilon}\left(t_{1}^{\varepsilon}, \tilde{x}^{\varepsilon}\left(t_{1}^{\varepsilon}\right), u_{1}\right)-\tilde{f}^{\varepsilon}\left(t_{1}^{\varepsilon}, \tilde{x}^{\varepsilon}\left(t_{1}^{\varepsilon}\right), u^{\varepsilon}\left(t_{1}^{\varepsilon}\right)\right) .
\end{aligned}
$$

Since $\tilde{f}^{\varepsilon}\left(t_{1}^{\varepsilon}, \tilde{x}^{\varepsilon}\left(t_{1}^{\varepsilon}\right), u^{\varepsilon}\left(t_{1}^{\varepsilon}\right)\right)$ converges to $\tilde{f}\left(t_{1}, \tilde{x}\left(t_{1}\right), u\left(t_{1}\right)\right)$, it follows that $\tilde{v}_{\pi^{\varepsilon}}\left(t_{1}^{\varepsilon}\right)$ converges to $\tilde{v}_{\pi}\left(t_{1}\right)$. From Proposition 2.6, $t_{f}^{\varepsilon}$ converges to $t_{f}, x^{\varepsilon}(\cdot)$ converges uniformly to $x(\cdot)$, and $\frac{\partial \tilde{f}^{\varepsilon}}{\partial \tilde{x}}\left(\cdot, \tilde{x}^{\varepsilon}(\cdot), u^{\varepsilon}(\cdot)\right)$ converges to $\frac{\partial \tilde{f}}{\partial \tilde{x}}(\cdot, \tilde{x}(\cdot), u(\cdot))$ for the weak star topology

\footnotetext{
${ }^{6}$ Note that $t_{1}^{\varepsilon}$ is a Lebesgue point of the function $t \mapsto \tilde{f}^{\varepsilon}\left(t, x^{\varepsilon}(t), u^{\varepsilon}(t)\right)$.
} 
of $L^{\infty}$, as $\varepsilon$ tends to 0 . Therefore, $\tilde{v}_{\pi^{\varepsilon}}^{\varepsilon}(\cdot)$ converges uniformly to $\tilde{v}_{\pi}(\cdot)$ as long as $x(t) \in X_{\alpha}(t)$ (see e.g. [37] for this kind of standard argument).

Note that Lemma 2.11 was used here to initialize the needle-like variation $u_{\pi^{\varepsilon}}^{\varepsilon}(\cdot)$ of the regularized control $u^{\varepsilon}(\cdot)$. The difficulty was that $u_{\pi^{\varepsilon}}^{\varepsilon}(\cdot)$ cannot be initialized at the time $t_{1}$ in general, since we do not know whether the simple convergence of $\tilde{f}^{\varepsilon}\left(t_{1}, \tilde{x}^{\varepsilon}\left(t_{1}\right), u^{\varepsilon}\left(t_{1}\right)\right)$ to $\tilde{f}\left(t_{1}, \tilde{x}\left(t_{1}\right), u\left(t_{1}\right)\right)$ holds or not, as $\varepsilon$ tends to 0 . This was the first main difficulty of the proof of Proposition 2.10 (already present in [33] but however easier to overcome in that reference).

The second main difficulty occurs when $x(\cdot)$ crosses $\partial X_{\alpha}(\cdot)$, since the variation vector $\tilde{v}_{\pi}(\cdot)$ has a jump (2.6) at the crossing time $t_{c}$. Our aim is to prove that, at the limit, we recover this jump for $\tilde{v}_{\pi^{\varepsilon}}(\cdot)$. Let us first prove the following technical lemma.

Lemma 2.12. The function $q(\cdot)$ defined by

$$
q(s)=\frac{\left\langle\left(\begin{array}{c}
\partial_{x} F(s, x(s)) \\
0
\end{array}\right), \tilde{v}_{\pi}(s)\right\rangle}{\left\langle\partial_{x} F(x, x(s)), f(s, x(s), u(s))\right\rangle+\partial_{t} F(x, x(s))}
$$

is continuous in a neighborhood of the crossing time $t_{c}$. For every $\varepsilon \in\left(0, \varepsilon_{0}\right)$, define

$$
q^{\varepsilon}(s)=\frac{\left\langle\left(\begin{array}{c}
\partial_{x} F\left(s, x^{\varepsilon}(s)\right) \\
0
\end{array}\right), \tilde{v}_{\pi}^{\varepsilon}(s)\right\rangle}{\left\langle\partial_{x} F\left(s, x^{\varepsilon}(s)\right), f^{\varepsilon}\left(s, x^{\varepsilon}(s), u^{\varepsilon}(s)\right)\right\rangle+\partial_{t} F\left(s, x^{\varepsilon}(s)\right)} .
$$

For every $s$ close to $t_{c}, q^{\varepsilon}(s)$ converges to $q(s)$ as $\varepsilon$ tends to 0 .

Proof. [Proof of Lemma 2.12] The first part of the lemma follows from the SnellDescartes like formula (2.7) established in Remark 2.1. For the second part, we proceed similarly as we did in Section 2.1 to derive the jump formula for the variation vector. Denote $\tilde{x}^{\varepsilon}(t, \eta)=\tilde{x}_{\pi^{\varepsilon}}^{\varepsilon}(t)$; one has $\tilde{x}^{\varepsilon}(t, \eta)=\left(x^{\varepsilon}(t, \eta), x^{0 \varepsilon}(t, \eta)\right)$. For every real number $\gamma$ such that $|\gamma|$ is small enough, denote by $t^{\varepsilon}(\eta, \gamma)$ (also denoted $t_{\eta, \gamma}^{\varepsilon}$ ) the first time at which $F\left(t_{\eta, \gamma}^{\varepsilon}, x^{\varepsilon}\left(t_{\eta, \gamma}^{\varepsilon}, \eta\right)\right)=\gamma$, and denote by $t(\eta, \gamma)$ (also denoted $t_{\eta, \gamma}$ ) the first time at which $F\left(t_{\eta, \gamma}, x\left(t_{\eta, \gamma}, \eta\right)\right)=\gamma$. Using the implicit function theorem, the transversality crossing assumption $\left(H_{4}\right)$ implies that, for $|\gamma|$ and $\varepsilon$ small enough, the function $\eta \mapsto t_{\eta, \gamma}^{\varepsilon}$ is derivable at 0 , and

$$
\begin{array}{r}
\left\langle\partial_{x} F\left(t_{0, \gamma}^{\varepsilon}, x^{\varepsilon}\left(t_{0, \gamma}^{\varepsilon}\right)\right), f^{\varepsilon}\left(t_{0, \gamma}^{\varepsilon}, x^{\varepsilon}\left(t_{0, \gamma}^{\varepsilon}\right), u^{\varepsilon}\left(t_{0, \gamma}^{\varepsilon}\right)\right) \frac{\partial t^{\varepsilon}}{\partial \eta}(0, \gamma)+v_{\pi^{\varepsilon}}^{\varepsilon}\left(t_{0, \gamma}^{\varepsilon}\right)\right\rangle \\
+\partial_{t} F\left(t_{0, \gamma}^{\varepsilon}, x^{\varepsilon}\left(t_{0, \gamma}^{\varepsilon}\right)\right) \cdot \frac{\partial t^{\varepsilon}}{\partial \eta}(0, \gamma)=0,
\end{array}
$$

and moreover the function $\gamma \mapsto t^{\varepsilon}(0, \gamma)$ converges to the function $\gamma \mapsto t(0, \gamma)$ in $C^{1}$ topology. Using again Assumption $\left(H_{4}\right)$, it is clear that the function $\gamma \mapsto t(0, \gamma)$, restricted to a neighborhood of 0 , is a diffeomorphism. The result follows, using the change of variable $s=t(0, \gamma)$. $\square$

To recover the jump formula as $\varepsilon$ tends to 0 , we next proceed again similarly as in Section 2.1. Denote as in the previous lemma $\tilde{x}^{\varepsilon}(t, \eta)=\tilde{x}_{\pi^{\varepsilon}}^{\varepsilon}(t)$. Let $t^{\varepsilon}(\eta)$ (also denoted $\left.t_{\eta}^{\varepsilon}\right)$ denote the first time at which $x^{\varepsilon}\left(t_{\eta}^{\varepsilon}, \eta\right) \in \partial X_{\alpha}\left(t_{\eta}^{\varepsilon}\right)$, that is, $F\left(t_{\eta}^{\varepsilon}, x^{\varepsilon}\left(t_{\eta}^{\varepsilon}, \eta\right)\right)=0$ (this corresponds to $\gamma=0$ in the previous proof). Using the transversality crossing assumption $\left(H_{4}\right)$ and the convergence of $x^{\varepsilon}(\cdot)$ to $x(\cdot)$, we get that the function $\eta \mapsto$ $t^{\varepsilon}(\eta)$ is derivable at $\eta=0$ for $\varepsilon>0$ small enough, and

$$
\left\langle\partial_{x} F\left(t_{0}^{\varepsilon}, x^{\varepsilon}\left(t_{0}^{\varepsilon}\right)\right), t^{\varepsilon^{\prime}}(0) \dot{x}^{\varepsilon}\left(t_{0}^{\varepsilon}\right)+\frac{\partial x^{\varepsilon}}{\partial \eta}\left(t_{0}^{\varepsilon}, 0\right)\right\rangle+t^{\varepsilon^{\prime}}(0) \partial_{t} F\left(t_{0}^{\varepsilon}, x^{\varepsilon}\left(t_{0}^{\varepsilon}\right)\right)=0,
$$


where $t_{0}^{\varepsilon}=t^{\varepsilon}(0)$, and hence, using Lemma 2.12,

$$
t^{\varepsilon^{\prime}}(0)=-q^{\varepsilon}(t)
$$

Let $\delta>0$. One has $\tilde{x}_{\pi^{\varepsilon}}^{\varepsilon}\left(t_{\eta}^{\varepsilon}+\delta\right)=\tilde{E}\left(\varepsilon, x_{\pi^{\varepsilon}}^{\varepsilon}\left(t_{\eta}^{\varepsilon}-\delta\right), 2 \delta, u_{\pi^{\varepsilon}}^{\varepsilon}(\cdot)_{\mid\left[t_{\eta}^{\varepsilon}-\delta, t_{\eta}^{\varepsilon}+\delta\right]}\right)$, and

$$
\tilde{x}_{\pi^{\varepsilon}}^{\varepsilon}\left(t_{\eta}^{\varepsilon}+\delta\right)=\tilde{E}\left(\varepsilon, \tilde{E}\left(\varepsilon, \tilde{x}_{\pi^{\varepsilon}}^{\varepsilon}\left(t_{\eta}^{\varepsilon}-\delta\right), \delta, u_{\pi^{\varepsilon}}^{\varepsilon}(\cdot)_{\mid\left[t_{\eta}^{\varepsilon}-\delta, t_{\eta}^{\varepsilon}\right]}\right), \delta, u_{\pi^{\varepsilon}}^{\varepsilon}(\cdot)_{\mid\left[t_{\eta}^{\varepsilon}, t_{\eta}^{\varepsilon}+\delta\right]}\right),
$$

for every $\eta>0$ small enough. On every piece, the end-point mapping is differentiable, and hence, since $\eta \mapsto t^{\varepsilon}(\eta)$ is derivable at 0 , we can derivate the above equality with respect to $\eta$, and take $\eta=0$. This yields

$$
\begin{aligned}
& \tilde{v}_{\pi^{\varepsilon}}^{\varepsilon}\left(t_{0}^{\varepsilon}+\delta\right)+t^{\varepsilon^{\prime}}(0) \dot{\tilde{x}}^{\varepsilon}\left(t_{0}^{\varepsilon}+\delta\right) \\
= & \frac{\partial \tilde{E}}{\partial x}\left(\varepsilon, \tilde{E}\left(\varepsilon, \tilde{x}^{\varepsilon}\left(t_{0}^{\varepsilon}-\delta\right), \delta, u^{\varepsilon}(\cdot)_{\mid\left[t_{0}^{\varepsilon}-\delta, t_{0}^{\varepsilon}\right]}\right), \delta, u^{\varepsilon}(\cdot)_{\mid\left[t_{0}^{\varepsilon}, t_{0}^{\varepsilon}+\delta\right]}\right) \\
& \circ \frac{\partial \tilde{E}}{\partial x}\left(\varepsilon, \tilde{x}^{\varepsilon}\left(t_{0}^{\varepsilon}-\delta\right), \delta, u^{\varepsilon}(\cdot)_{\mid\left[t_{0}^{\varepsilon}-\delta, t_{0}^{\varepsilon}\right]}\right) \cdot\left(t^{\varepsilon^{\prime}}(0) \dot{\tilde{x}}^{\varepsilon}\left(t_{0}^{\varepsilon}-\delta\right)+\tilde{v}_{\pi^{\varepsilon}}^{\varepsilon}\left(t_{0}^{\varepsilon}-\delta\right)\right) .
\end{aligned}
$$

We first let $\varepsilon$ tends to 0 , and then, let $\delta$ tends to 0 . Using (2.16) and Lemma 2.12, we infer that

$$
\lim _{\delta \rightarrow 0} \lim _{\varepsilon \rightarrow 0}\left(\tilde{v}_{\pi^{\varepsilon}}^{\varepsilon}\left(t_{0}^{\varepsilon}+\delta\right)-\tilde{v}_{\pi^{\varepsilon}}^{\varepsilon}\left(t_{0}^{\varepsilon}-\delta\right)\right)=q(t)\left(\tilde{f}_{\beta}\left(t^{+}\right)-\tilde{f}_{\alpha}\left(t^{-}\right)\right),
$$

which is corresponds exactly to the formula (2.6). Hence, we have proved that

$$
\lim _{\delta \rightarrow 0} \lim _{\varepsilon \rightarrow 0}\left(\tilde{v}_{\pi^{\varepsilon}}^{\varepsilon}\left(t^{\varepsilon}(0)+\delta\right)-\tilde{v}_{\pi^{\varepsilon}}^{\varepsilon}\left(t^{\varepsilon}(0)-\delta\right)\right)=\tilde{v}_{\pi}\left(t_{c}^{+}\right)-\tilde{v}_{\pi}\left(t_{c}^{-}\right) .
$$

This formula shows that, when $x(\cdot) \operatorname{crosses} \partial X_{\alpha}(\cdot)$ and the variation vector $\tilde{v}_{\pi}(\cdot)$ has a jump (2.6) at the crossing time $t_{c}$, we recover this jump at the limit for $\tilde{v}_{\pi^{\varepsilon}}(\cdot)$. Notice that, of course, $\tilde{v}_{\pi^{\varepsilon}}(\cdot)$ is continuous, and the order of the limits in (2.17) cannot be switched.

We can end the proof of the proposition. Indeed, before the first crossing time, $\tilde{v}_{\pi^{\varepsilon}}(\cdot)$ converges uniformly to $\tilde{v}_{\pi}(\cdot)$ on every compact interval not containing this crossing time. At the crossing time, $\tilde{v}_{\pi^{\varepsilon}}(\cdot)$ has a jump, and the formula (2.17) shows that we recover this jump, at the limit, for $\tilde{v}_{\pi^{\varepsilon}}(\cdot)$. Then, beyond the first crossing time and before the second crossing time, one has uniform convergence as well, and the argument goes by iteration.

REMARK 2.13. The same convergence result holds for the pontryagin cone $K_{1}$. We will also need the following statement, resulting obviously from the above proof: for every $t \in\left[0, t_{f}\right]$, for every $\tilde{v} \in \tilde{K}_{1}(t)$, for every $\varepsilon>0$ there exists $\tilde{v}^{\varepsilon} \in \tilde{K}_{1}^{\varepsilon}(t)$ such that $\tilde{v}^{\varepsilon}$ converges to $\tilde{v}$ as $\varepsilon$ tends to 0 .

Proposition 2.14. There exists $\varepsilon_{0}>0$ such that, for every $\varepsilon \in\left(0, \varepsilon_{0}\right)$, every extremal lift $\left(x^{\varepsilon}(\cdot), p^{\varepsilon}(\cdot), p^{0 \varepsilon}, u^{\varepsilon}(\cdot)\right)$ of any solution $x^{\varepsilon}(\cdot)$ of $(\boldsymbol{O C P})_{\varepsilon}$ is normal. Furthermore, setting $p^{0 \varepsilon}=-1$, the set $\left\{p^{\varepsilon}(t) \mid t \in\left[0, t_{f}^{\varepsilon}\right], 0<\varepsilon<\varepsilon_{0}\right\}$ is bounded.

Proof. We argue by contradiction. Assume that, for every integer $k$, there exist $\varepsilon_{k} \in(0,1 / n)$ and a solution $x^{\varepsilon_{k}}(\cdot)$ of $(\mathbf{O C P})_{\varepsilon_{k}}$ having an abnormal extremal lift $\left(x^{\varepsilon_{k}}(\cdot), p^{\varepsilon_{k}}(\cdot), 0, u^{\varepsilon_{k}}(\cdot)\right)$. Set $\psi^{\varepsilon_{k}}=p^{\varepsilon_{k}}\left(t_{f}^{\varepsilon_{k}}\right)$, for every integer $k$. Then, from Remark 2.4, one has $\left\langle\psi^{\varepsilon_{k}}, v^{\varepsilon_{k}}\right\rangle \leqslant 0$, for every $v^{\varepsilon_{k}} \in K^{\varepsilon_{k}}\left(t_{f}^{\varepsilon_{k}}\right)$, and, since the final time is free, $M\left(\varepsilon_{k}\right)=\max _{w \in \Omega}\left\langle\psi^{\varepsilon_{k}}, f^{\varepsilon}\left(t_{f}^{\varepsilon_{k}}, x^{\varepsilon_{k}}\left(t_{f}^{\varepsilon_{k}}\right), w\right)\right\rangle=0$, for every integer $k$. Since the final adjoint vector $\left(p^{\varepsilon_{k}}\left(t_{f}^{\varepsilon_{k}}\right), p^{0 \varepsilon_{k}}\right)$ (here, $\left.p^{0 \varepsilon_{k}}=0\right)$ is defined up to a multiplicative scalar, 
we assume that $\psi^{\varepsilon_{k}}$ is a unit vector for every integer $k$. Then, up to a subsequence, the sequence $\left(\psi^{\varepsilon_{k}}\right)_{k \in \mathbb{N}}$ converges to some unit vector $\psi \in \mathbb{R}^{n}$.

In order to pass to the limit, we need the following easy lemma.

LEMma 2.15. Let $m$ be a positive integer, $g$ be a continuous function on $\mathbb{R} \times \mathbb{R}^{m}$, and $C$ be a compact subset of $\mathbb{R}^{m}$. For every $\varepsilon>0$, set $M(\varepsilon)=\max _{u \in C} g(\varepsilon, u)$, and $M=\max _{u \in C} g(0, u)$. Then, $M(\varepsilon)$ tends to $M$ as $\varepsilon$ tends to 0 .

Proof. For every $\varepsilon>0$, let $u_{\varepsilon} \in C$ such that $M(\varepsilon)=g\left(\varepsilon, u_{\varepsilon}\right)$, and let $u \in C$ such that $M=g(0, u)$. Note that $u_{\varepsilon}$ does not necessarily converge to $u$, however we will prove that $M(\varepsilon)$ tends to $M$, as $\varepsilon$ tends to 0 . Let $u_{0} \in C$ be a closure point of the family $\left(u_{\varepsilon}\right)_{\varepsilon>0}$. Then, by definition of $M$, one has $g\left(0, u_{0}\right) \leqslant M$. On the other hand, since $g$ is continuous, $g(\varepsilon, u)$ tends to $g(0, u)=M$ as $\varepsilon$ tends to 0 . By definition, $g(\varepsilon, u) \leqslant M(\varepsilon)=g\left(\varepsilon, u_{\varepsilon}\right)$ for every $\varepsilon>0$. Therefore, passing to the limit, one gets $M \leqslant g\left(0, u_{0}\right)$. It follows that $M=g\left(0, u_{0}\right)$. We have thus proved that the (bounded) family $(M(\varepsilon))_{\varepsilon>0}$ of real numbers has a unique closure point, which is $M$. The conclusion follows.

Using Proposition 2.6, Lemma 2.15, and Proposition 2.10, passing to the limit we infer that $\langle\psi, v\rangle \leqslant 0$ for every $v \in K\left(t_{f}\right)$ and that $M=\max _{w \in \Omega}\left\langle\psi, f\left(t_{f}, x\left(t_{f}\right), w\right)\right\rangle=$ 0 . It then follows from Remark 2.4 that the trajectory $x(\cdot)$ has an abnormal extremal lift. This contradicts Assumption $\left(H_{3}\right)$.

To derive the second part of the statement of the proposition, let us first prove the following lemma.

Lemma 2.16. Setting $p^{0 \varepsilon}=-1$, the set of all possible $p^{\varepsilon}\left(t_{f}^{\varepsilon}\right)$, with $\varepsilon \in\left(0, \varepsilon_{0}\right)$, is bounded.

Proof. [Proof of Lemma 2.16] We proceed again by contradiction. Assume that there exists a sequence $\left(\varepsilon_{k}\right)_{k \in \mathbb{N}}$ of positive real numbers converging to 0 such that $\left\|p^{\varepsilon_{k}}\left(t_{f}^{\varepsilon_{k}}\right)\right\|$ tends to $+\infty$. Since the sequence $\left(\frac{p^{\varepsilon_{k}}\left(t_{f}^{\varepsilon_{k}}\right)}{\left\|p^{\varepsilon_{k}}\left(t_{f}^{\varepsilon_{k}}\right)\right\|}\right)_{k \in \mathbb{N}}$ is bounded in $\mathbb{R}^{n}$, up to a subsequence it converges to some unit vector $\psi$. Using the Lagrange multipliers property and (2.11), there holds $\left\langle p^{\varepsilon_{k}}\left(t_{f}^{\varepsilon_{k}}\right), v^{\varepsilon_{k}}\right\rangle \leqslant 0$ for every $v^{\varepsilon_{k}} \in K^{\varepsilon_{k}}\left(t_{f}^{\varepsilon_{k}}\right)$, and

$$
\max _{w \in \Omega}\left(\left\langle p^{\varepsilon_{k}}\left(t_{f}^{\varepsilon_{k}}\right), f^{\varepsilon_{k}}\left(t_{f}^{\varepsilon_{k}}, x^{\varepsilon_{k}}\left(t_{f}^{\varepsilon_{k}}\right), w\right)\right\rangle-f^{0 \varepsilon_{k}}\left(t_{f}^{\varepsilon_{k}}, x^{\varepsilon_{k}}\left(t_{f}^{\varepsilon_{k}}\right), w\right)\right)=0,
$$

for every integer $k$. Dividing by $\left\|p^{\varepsilon_{k}}\left(t_{f}^{\varepsilon_{k}}\right)\right\|$, and passing to the limit, using Proposition 2.6, Lemma 2.15, Proposition 2.10 and Remark 2.4, the same reasoning as above yields that the trajectory $x(\cdot)$ has an abnormal extremal lift, which is a contradiction. $\square$

We now use the fact that, for every $\varepsilon \in\left(0, \varepsilon_{0}\right)$, the function $t \mapsto\left\langle\tilde{p}^{\varepsilon}(t), \tilde{v}_{\pi^{\varepsilon}}^{\varepsilon}(t)\right\rangle$ is constant $^{7}$, for every variation vector $\tilde{v}_{\pi^{\varepsilon}}(\cdot)$ along $x^{\varepsilon}(\cdot)$, where $\tilde{p}^{\varepsilon}(t)=\left(p^{\varepsilon}(t),-1\right)$. In particular, denoting $\tilde{v}_{\pi^{\varepsilon}}^{\varepsilon}(t)=\left(v_{\pi^{\varepsilon}}^{\varepsilon}(t), v_{\pi^{\varepsilon}}^{0 \varepsilon}(t)\right)$, this yields

$$
\left\langle p^{\varepsilon}(t), v_{\pi^{\varepsilon}}^{\varepsilon}(t)\right\rangle-v_{\pi^{\varepsilon}}^{0 \varepsilon}(t)=\left\langle p^{\varepsilon}\left(t_{f}^{\varepsilon}\right), v_{\pi^{\varepsilon}}^{\varepsilon}\left(t_{f}^{\varepsilon}\right)\right\rangle-v_{\pi^{\varepsilon}}^{0 \varepsilon}\left(t_{f}^{\varepsilon}\right),
$$

for every $t \in\left[0, t_{f}^{\varepsilon}\right]$. Since $\tilde{K}_{1}^{\varepsilon}\left(t_{f}^{\varepsilon}\right)$ is a cone, we assume that the variation vectors under consideration here are such that $\tilde{v}_{\pi^{\varepsilon}}^{\varepsilon}\left(t_{f}^{\varepsilon}\right)$ is a unit vector of $\mathbb{R}^{n+1}$. In particular, using such variation vectors, Lemma 2.16 implies that the right-hand side of (2.19) remains uniformly bounded with respect to $\varepsilon$. Since the component $v_{\pi^{\varepsilon}}^{0 \varepsilon}(\cdot)$ satisfies

$$
\dot{v}_{\pi^{\varepsilon}}^{0 \varepsilon}(t)=\frac{\partial f^{0 \varepsilon}}{\partial x}\left(t, x^{\varepsilon}(t), u^{\varepsilon}(t)\right) v_{\pi^{\varepsilon}}^{\varepsilon}(t)
$$

\footnotetext{
${ }^{7}$ Indeed, it is continuous and its derivative is everywhere equal to zero.
} 
for almost every $t \in\left[0, t_{f}^{\varepsilon}\right]$, and since, from Proposition 2.6, $\frac{\partial f^{0 \varepsilon}}{\partial x}\left(\cdot, x^{\varepsilon}(\cdot), u^{\varepsilon}(\cdot)\right)$ converges to $\frac{\partial f^{0}}{\partial x}(\cdot, x(\cdot), u(\cdot))$ for the weak star topology of $L^{\infty}$ as $\varepsilon$ tends to 0 , it follows easily that the set of all $v_{\pi^{\varepsilon}}^{0 \varepsilon}(t)$ under consideration, for $t \in\left[0, t_{f}^{\varepsilon}\right]$ and $0<\varepsilon<\varepsilon_{0}$, is bounded. Therefore, we infer from (2.19) that the set of all $\left\langle p^{\varepsilon}(t), v_{\pi^{\varepsilon}}^{\varepsilon}(t)\right\rangle$, over all possible variation vectors such that $\tilde{v}_{\pi^{\varepsilon}}^{\varepsilon}\left(t_{f}^{\varepsilon}\right)$ is a unit vector, is uniformly bounded with respect to $\varepsilon$. From Assumption $\left(H_{3}\right)$ and Lemma 2.5, there holds $K_{1}\left(t_{f}\right)=\mathbb{R}^{n}$, and therefore, from Proposition 2.10, there holds $K_{1}^{\varepsilon}\left(t_{f}^{\varepsilon}\right)=\mathbb{R}^{n}$ for $\varepsilon>0$ small enough; hence the previous arguments imply that the set of all $p^{\varepsilon}(t)$ for $t \in\left[0, t_{f}^{\varepsilon}\right]$ is uniformly bounded with respect to $\varepsilon$.

REMARK 2.17. If we remove the assumption that the optimal trajectory $x(\cdot)$ has a unique extremal lift, which is moreover normal, then Proposition 2.14 still holds provided that every extremal lift of $x(\cdot)$ is normal.

We are now in a position to end the proof of the theorems and to derive the convergence properties for the adjoint vector.

Recall that $(x(\cdot), p(\cdot),-1, u(\cdot))$ is the unique (normal) extremal lift of $x(\cdot)$, the solution of (HOCP), and that, for every $\varepsilon \in\left(0, \varepsilon_{0}\right),\left(x^{\varepsilon}(\cdot), p^{\varepsilon}(\cdot),-1, u^{\varepsilon}(\cdot)\right)$ is a (normal) extremal lift of a solution $x^{\varepsilon}(\cdot)$ of $(\mathbf{O C P})_{\varepsilon}$. Let us prove that $p^{\varepsilon}(\cdot)$ converges uniformly to $p(\cdot)$ on every closed subinterval of $\left[0, t_{f}\right]$ that does not contain any crossing time, as $\varepsilon$ tends to 0 . For every $\varepsilon \in\left(0, \varepsilon_{0}\right)$, set $\psi^{\varepsilon}=p^{\varepsilon}\left(t_{f}^{\varepsilon}\right)$. Recall that $p^{\varepsilon}(\cdot)$ is solution of the Cauchy problem

$$
\dot{p}^{\varepsilon}(t)=-\left\langle p^{\varepsilon}(t), \frac{\partial f^{\varepsilon}}{\partial x}\left(t, x^{\varepsilon}(t), u^{\varepsilon}(t)\right)\right\rangle-\frac{\partial f^{0 \varepsilon}}{\partial x}\left(t, x^{\varepsilon}(t), u^{\varepsilon}(t)\right), \quad p^{\varepsilon}\left(t_{f}^{\varepsilon}\right)=\psi^{\varepsilon},
$$

for almost every $t \in\left[0, t_{f}^{\varepsilon}\right]$, and moreover, $\left\langle\psi^{\varepsilon}, v^{\varepsilon}\right\rangle \leqslant 0$ for every $v^{\varepsilon} \in K^{\varepsilon}\left(t_{f}^{\varepsilon}\right)$, and $\max _{w \in \Omega}\left(\left\langle\psi^{\varepsilon}, f^{\varepsilon}\left(t_{f}^{\varepsilon}, x^{\varepsilon}\left(t_{f}^{\varepsilon}\right), w\right)\right\rangle-f^{0 \varepsilon}\left(t_{f}^{\varepsilon}, x^{\varepsilon}\left(t_{f}^{\varepsilon}\right), w\right)\right)=0$, for every $\varepsilon \in\left(0, \varepsilon_{0}\right)$. From Proposition 2.14, the family $\left(\psi^{\varepsilon}\right)_{0<\varepsilon<\varepsilon_{0}}$ is bounded; let $\psi$ be a closure point of that family and $\left(\varepsilon_{k}\right)_{k \in \mathbb{N}}$ a sequence of positive real numbers converging to 0 such that $\psi^{\varepsilon_{k}}$ tends to $\psi$. Using Proposition 2.6, and as in the proof of this result, we infer that the sequence $\left(p^{\varepsilon_{k}}(\cdot)\right)_{k \in \mathbb{N}}$ converges uniformly to the solution $z(\cdot)$ of the Cauchy problem

$$
\dot{z}(t)=-\left\langle z(t), \frac{\partial f_{\beta}}{\partial x}(t, x(t), u(t))\right\rangle-\frac{\partial f_{\beta}^{0}}{\partial x}(t, x(t), u(t)), \quad z\left(t_{f}\right)=\psi,
$$

for almost every $t$ belonging to some interval $\left[\tau, t_{f}\right]$, provided that the interval $\left[\tau, t_{f}\right]$ does not contain any crossing time (and assuming that $x(t) \in X_{\beta}(t)$ for every $t \in$ $\left.\left[\tau, t_{f}\right]\right)$. Moreover, passing to the limit as in the previous proof, one gets $\langle\psi, v\rangle \leqslant 0$ for every $v \in K\left(t_{f}\right)$, and $\max _{w \in \Omega}\left(\left\langle\psi, f_{\beta}\left(t_{f}, x\left(t_{f}\right), w\right)\right\rangle-f_{\beta}^{0}\left(t_{f}, x\left(t_{f}\right), w\right)\right)=0$. It follows that $(x(\cdot), z(\cdot),-1, u(\cdot))$ is an extremal lift of $x(\cdot)$ on the interval $\left[\tau, t_{f}\right]$, and from Assumption $\left(H_{3}\right)$ we infer that $z(\cdot)$ and $p(\cdot)$ coincide on the subinterval $\left[\tau, t_{f}\right]$.

We can proceed in reverse time in such a way as long as we do not encounter any crossing time. In order to take into account possible crossing times, we must use an additional argument. Let $t$ be an element of $\left[0, t_{f}\right]$ that is not a crossing time. From Proposition 2.14, the family $\left(p^{\varepsilon}(t)\right)_{0<\varepsilon<\varepsilon_{0}}$ is bounded and hence has a closure point $\bar{p}(t)$. To end the proof, it suffices to prove that $\bar{p}(t)=p(t)$ (indeed, one this fact is proved, the uniform convergence argument is similar to the one above). From Remark 2.13, for every variation vector $\tilde{v}_{\pi}(t) \in \tilde{K}_{1}(t)$, for every $\varepsilon>0$ there exists $\tilde{v}_{\pi^{\varepsilon}}^{\varepsilon}(t) \in \tilde{K}_{1}(t)$ such that $\tilde{v}_{\pi^{\varepsilon}}^{\varepsilon}(t)$ converges to $\tilde{v}_{\pi}(t)$ as $\varepsilon$ tends to 0 . Setting $\tilde{p}^{\varepsilon}=\left(p^{\varepsilon},-1\right)$, as in the proof of Lemma 2.16 we use the fact that the function 
$t \mapsto\left\langle\tilde{p}^{\varepsilon}(t), \tilde{v}_{\pi^{\varepsilon}}^{\varepsilon}(t)\right\rangle$ is constant and this yields Equation (2.19) at the time $t$. Passing to the limit, we get

$$
\left\langle\bar{p}(t), v_{\pi}(t)\right\rangle-v_{\pi}^{0}(t)=\left\langle p\left(t_{f}\right), v_{\pi}\left(t_{f}\right)\right\rangle-v_{\pi}^{0}\left(t_{f}\right) .
$$

In order to prove that $\bar{p}(t)=p(t)$, we first need to derive the following lemma, which is an extension to the hybrid context of the well known result that we used formerly.

Lemma 2.18. Denoting $\tilde{p}(t)=(p(t),-1)$, the function $t \mapsto\left\langle\tilde{p}(t), \tilde{v}_{\pi}(t)\right\rangle$ is constant on $\left[0, t_{f}\right]$, for every variation vector $\tilde{v}_{\pi}(\cdot)$.

Proof. [Proof of Lemma 2.18] It is easy to see that the derivative of the function $t \mapsto\left\langle\tilde{p}(t), \tilde{v}_{\pi}(t)\right\rangle$ is equal to 0 everywhere, using the differential equations satisfied by $\tilde{p}(\cdot)$ and $\tilde{v}_{\pi}(\cdot)$. Besides, this function is clearly continuous outside the crossing times. Hence, to prove the statement it suffices to see that the function remains continuous at crossing times. But this follows straightforwardly from a simple computation using the jump conditions (1.5) and (2.6).

It follows from this lemma that $\left\langle p(t), v_{\pi}(t)\right\rangle-v_{\pi}^{0}(t)=\left\langle p\left(t_{f}\right), v_{\pi}\left(t_{f}\right)\right\rangle-v_{\pi}^{0}\left(t_{f}\right)$, and hence, we infer from 2.21 that $\left\langle\bar{p}(t), v_{\pi}(t)\right\rangle=\left\langle p(t), v_{\pi}(t)\right\rangle$. Since this equality holds for every variation vector, and since $K_{1}(t)=\mathbb{R}^{n}$ (this follows from Assumption $\left(H_{3}\right)$ and Lemma 2.5), it follows that $\bar{p}(t)=p(t)$. This ends the proof of the theorems.

REMARK 2.19. In the proof above, it is possible to replace Assumptions $\left(\mathrm{H}_{2}\right)$ and $\left(\mathrm{H}_{3}\right)$ with the weaker assumption that every extremal lift of every solution of (HOCP) is normal. In that case, using the same arguments, we prove that every closure point of the family $\left(t_{f}^{\varepsilon}, x^{\varepsilon}(\cdot), \dot{x}^{\varepsilon}(\cdot), p^{\varepsilon}(\cdot)\right)_{0<\varepsilon<\varepsilon_{0}}$ (for the evident topologies) can be written as $(T, \bar{x}(\cdot), \dot{\bar{x}}(\cdot), \bar{p}(\cdot))$, where $\bar{x}(\cdot)$ is solution of (1.1) associated with a control $\bar{u}(\cdot) \in L^{\infty}\left(\left[0, t_{f}\right], \Omega\right)$, such that $\bar{x}(0) \in M_{0}$ and $\bar{x}\left(t_{f}\right) \in M_{1}$. Moreover, $(\bar{x}(\cdot), \bar{u}(\cdot))$ is solution of $(\boldsymbol{H O C P})$ defined on $[0, T]$, having as a normal extremal lift the 4-tuple $(\bar{x}(\cdot), \bar{p}(\cdot),-1, \bar{u}(\cdot))$.

\section{Application to the minimal time low-thrust coplanar orbit transfer with eclipse constraint.}

3.1. Problem statement. We focus on the coplanar orbit transfer of a satellite around the Earth. The satellite is modeled as a mass point and is assumed to evolve in a central gravitational field. We neglect the gravitational perturbations such as the Earth oblateness. The satellite follows the two-dimensional controlled Kepler equation

$$
\ddot{q}(t)=-\frac{\mu}{r(t)^{3}} q(t)+\frac{T(t)}{m(t)}, \quad \dot{m}(t)=-\beta\|T(t)\|,
$$

where $q(t) \in \mathbb{R}^{2}$ denotes the cartesian coordinates of the satellite in an inertial geocentric reference frame, $r(t)=\|q(t)\|$ is the distance to the Earth's center, $T(t) \in$ $\bar{B}\left(0, T_{\max }\right) \subset \mathbb{R}^{2}$ is the thrust, $T_{\max }$ is the maximal allowed thrust and $m(t)$ is the mass, $\mu$ stands for the Earth gravitational constant and $\beta$ is a coefficient depending on the thruster characteristics.

The objective is to realize a minimal time orbit transfer, for instance from a low or/and highly eccentric initial orbit to a geostationary final one. The controllability aspects of that problem were studied in $[4,5,8,18]$.

We are interested in low-thrust engines, that is with maximum thrust $T_{\max }$ small when compared with the mass of the satellite. Thus, the orbit transfer will require a lot of revolutions and the cartesian coordinates are not well suited. Indeed, the evolution of those coordinates is large when compared to the small evolution of the 
orbit shape. We use the set $x=\left(P, e_{x}, e_{y}, L\right)$ of Gauss coordinates defined by

$$
e_{x}=e \cos (\omega), e_{y}=\sin (\omega), L=\omega+\nu
$$

where $P$ is the semi-latus rectum of the osculating ellipsis, $e$ the eccentricity, $\nu$ the true anomaly and $\omega$ the argument of perigee. The osculating ellipsis is the ellipsis the satellite would follow if it were subject to no other forces than the central gravitational field. In those coordinates, the cartesian position $q$ and velocity $\dot{q}$ are given by

$$
q=\frac{P}{W}\left(\begin{array}{c}
\cos L \\
\sin L
\end{array}\right), \quad \dot{q}=\frac{\mu}{P}\left(\begin{array}{c}
-e_{y}-\sin L \\
e_{x}+\cos L
\end{array}\right),
$$

where $W=1+e_{x} \cos L+e_{y} \sin L$. In addition to this change of coordinates, we express the thrust $T$ in the moving reference frame $(q /\|q\|, s)$, where $s$ is the unit projection of $\dot{q}$ on the orthogonal of $q$. We also rewrite the control as $T(\cdot)=T_{\max } u(\cdot)$, where $u(\cdot)$ takes its values in the closed unit ball $\bar{B}(0,1)$ of $\mathbb{R}^{2}$. The equations of motion can then be rewritten as

$$
\dot{x}(t)=f_{0}(x(t))+\frac{T_{\max }}{m(t)}\left(u_{1}(t) f_{1}(x(t))+u_{2}(t) f_{2}(x(t))\right), \quad \dot{m}(t)=-\beta T_{\max }\|u(t)\|,
$$

where the vector fields $f_{0}, f_{1}$ and $f_{2}$ are defined by

$$
f_{0}(x)=\sqrt{\frac{\mu}{P}}\left(\begin{array}{c}
0 \\
0 \\
0 \\
\frac{W^{2}}{P}
\end{array}\right), f_{1}(x)=\sqrt{\frac{P}{\mu}}\left(\begin{array}{c}
0 \\
\sin L \\
\cos L \\
0
\end{array}\right), f_{2}(x)=\sqrt{\frac{P}{\mu}}\left(\begin{array}{c}
2 P / W \\
\cos L+\frac{e_{x}+\cos L}{W} \\
\sin L+\frac{e_{y}+\sin L}{W} \\
0
\end{array}\right) .
$$

We denote by $y=(x, m)$ the full state, and its dynamic will be written as $\dot{y}(t)=$ $f(y(t), u(t))$.

With no additional constraints, this optimal control problem has already been widely studied (see for instance $[4,5,8]$ ). We propose here to add a constraint and use the constrained problem to illustrate the convergence properties of our regularization approach.

The low-thrust orbit transfer is achieved thanks to electro-ionic thrusters that in practice cannot operate without any source of power. If this source of power is simply the sun, then the satellite is not actuated while standing in the shadow cone of the Earth. Considering the distance Sun-Earth and Earth-satellite, we can assume the shadow cone of the Earth to be a half cylinder (see Figure 3.1).

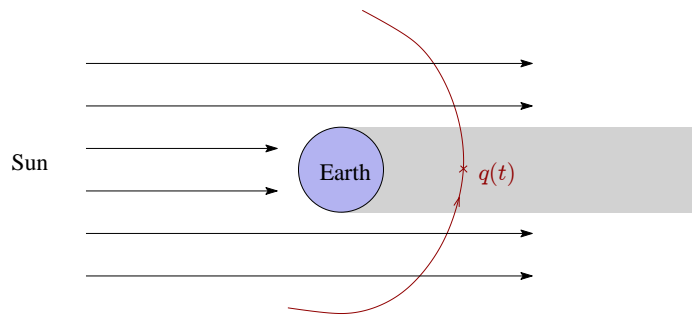

FIG. 3.1. Eclipse phenomenon and shadow cone 
This problem has already been studied in [15] with a regularization approach but without the present theoretical background regarding convergence and hybrid necessary conditions.

Denote by $\Omega_{c}(t)$ the inclination of the shadow cone in the geocentric inertial reference frame. The frontier between light and shadow is given by the zeros of the function $F_{r_{E}}$ defined by

$$
F_{r_{E}}(t, x)=\sin ^{2}\left(L-\Omega_{c}\right)-\left(\frac{r_{E} W}{P}\right)^{2}
$$

if $\cos \left(L-\Omega_{c}\right)>0$, where $r_{E}$ is the Earth radius. Note that we only need to consider this function in the neighborhood of the frontier. If we further assume the Earth orbit to be circular, then:

$$
\Omega_{c}(t)=\Omega_{c 0}+\frac{2 \pi}{\text { one year }} t
$$

where $\Omega_{c 0}$ is the inclination of the shadow cone at the initial time $t_{0}=0$.

3.2. Hybridization of the problem. The optimal control problem settled above is naturally a (HOCP). Instead of considering the control $u(\cdot)$ to be equal to zero in the shadow cone, we rather use a model with discontinuous vector fields $f_{1}$ and $f_{2}$, deciding that they are equal to zero in the shadow cone. With such a model, (HOCP) is written as follows. Let $\bar{f}_{0}$ be the vector field on $\mathbb{R}^{5}$ defined by $\bar{f}_{0}(y)=\left(f_{0}(x), 0\right)$. For every time $t$, denote by $X_{0}(t)$ the shadow cone and by $X_{1}(t)$ its complement. Then, we seek a trajectory $y(\cdot)=(x(\cdot), m(\cdot))$ solution on $\left[0, t_{f}\right]$ of the hybrid control system

$$
\dot{y}(t)=\left\{\begin{array}{lll}
f(y(t), u(t)) & \text { if } & x(t) \in X_{1}(t) \subset \mathbb{R}^{4}, \\
\bar{f}_{0}(y(t)) & \text { if } & x(t) \in X_{0}(t) \subset \mathbb{R}^{4},
\end{array}\right.
$$

associated with a control $u(\cdot)$ satisfying the constraint $\|u(\cdot)\| \leqslant 1$, starting from the initial conditions $P(0)=P_{0}, e_{x}(0)=e_{x 0}, e_{y}(0)=e_{y 0}, L(0)=L_{0}$, and joining in minimal time $t_{f}$ the final conditions $P\left(t_{f}\right)=P_{f}, e_{x}\left(t_{f}\right)=e_{x f}, e_{y}\left(t_{f}\right)=e_{y f}$. Note that $L\left(t_{f}\right)$ and $m\left(t_{f}\right)$ are free.

REMARK 3.1. Note that the partition of the state space only depends on the position variable $q$. This implies that the control vector fields $f_{1}$ and $f_{2}$ are orthogonal to the boundary at a crossing point.

From the HMP recalled in Section 1, every minimizing trajectory $y(\cdot)$ is the projection of an extremal $\left(y(\cdot), p(\cdot), p^{0}, u(\cdot)\right)$ satisfying (1.3) and (1.4), and the maximized Hamiltonian vanishes at the final time $t_{f}$. The adjoint vector has a jump (1.5) every time the trajectory crosses (transversally) a boundary. Denoting $p=$ $\left(p_{P}, p_{e_{x}}, p_{e_{y}}, p_{L}, p_{m}\right)$, notice that the component $p_{m}(\cdot)$ is continuous since the boundary only depends on $x$. Besides, one has the transversality conditions $p_{L}\left(t_{f}\right)=0$, $p_{m}\left(t_{f}\right)=0$.

REMARK 3.2. Due to a large number of revolutions, and hence of crossing times, it can be expected that the application of a shooting method to this problem, using the above necessary conditions, is difficult to carry out successfully. Indeed, we observe on our numerical simulations that the domain of convergence of the method is very small. This motivates the use of our regularization procedure, as explained next. Observe however that the usual orbit transfer problem in the absence of a shadow cone constraint has been solved by shooting methods (see e.g. [4, 8, 17, 18, 24]). 
3.3. Regularization of the hybrid optimal control problem. Our aim is to solve this problem using a shooting method but, as explained formerly, directly trying to find a zero of the shooting function associated with (HOCP) fails in general. The idea is then to define a regularization $(\mathbf{O C P})=$ of (HOCP) such that the solving of $(\mathbf{O C P})_{\varepsilon}$ using a single shooting method is easier than for (HOCP). Then, using a finite decreasing sequence of positive $\varepsilon_{i}$, we use the solution of $(\mathbf{O C P})_{\varepsilon_{i}}$ to initialize the solving of $(\mathbf{O C P})_{\varepsilon_{\mathbf{i}+1}}$. This iteration is performed until $\varepsilon_{i}$ is small enough and the last solution is used to initialize the solving of (HOCP). This process is justified by the convergence properties stated in our main results.

We define a regularization of the original (HOCP) by thickening the light / shadow frontier as in [15]. The procedure is the following. Define an imaginary planet, with the same center than the Earth and with radius $r_{\varepsilon}=(1-\varepsilon) r_{E}, \varepsilon \in[0,1)$. Figure 3.2 shows the two planets and their shadows. The plane $\mathbb{R}^{2}$ consists of three domains

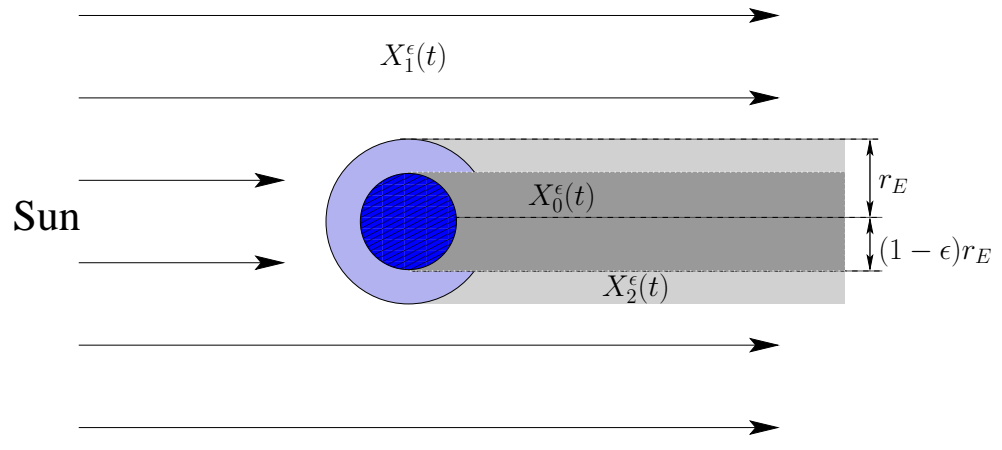

FIG. 3.2. The two planets and their shadow cones.

$X_{1}^{\varepsilon}(t), X_{0}^{\varepsilon}(t)$ and $X_{2}^{\varepsilon}(t)$, where $X_{1}^{\varepsilon}(t)$ denotes the domain completely exposed to the sun, $X_{0}^{\varepsilon}(t)$ the imaginary shadow cone and $X_{2}^{\varepsilon}(t)$ is the part of $\mathbb{R}^{2}$ that is in the shadow cone of the Earth but not in the imaginary shadow cone.

The regularized system is defined as

$$
\begin{aligned}
\dot{x}^{\varepsilon}(t) & =f_{0}^{\varepsilon}\left(x^{\varepsilon}(t)\right)+\frac{T_{\max }}{m(t)} b\left(\varepsilon, t, x^{\varepsilon}(t)\right)\left(u_{1}^{\varepsilon}(t) f_{1}\left(x^{\varepsilon}(t)\right)+u_{2}^{\varepsilon}(t) f_{2}\left(x^{\varepsilon}(t)\right)\right), \\
\dot{m}^{\varepsilon}(t) & =-\beta T_{\max } b\left(\varepsilon, t, x^{\varepsilon}(t)\right)\left\|u^{\varepsilon}(t)\right\|,
\end{aligned}
$$

where the function $b(\cdot, \cdot, \cdot)$, defined hereafter, is such that $b(\varepsilon, t, \cdot)$ is equal to 1 in $X_{1}^{\varepsilon}(t)$ and is equal to 0 in $X_{0}^{\varepsilon}(t)$. We define $b(\varepsilon, t, \cdot)$ as a fifth-order polynomial based on the Euclidean distance from $x(t)$ to one of the frontiers of the shadow cones, equal to 1 on the boundary between $X_{1}^{\varepsilon}(t)$ and $X_{2}^{\varepsilon}(t)$, equal to 0 on the boundary between $X_{0}^{\varepsilon}(t)$ and $X_{1}^{\varepsilon}(t)$, and such that its first and second derivatives with respect to $x$ vanish on both boundaries. Simple geometric arguments and computations lead to

$$
b(\varepsilon, t, x)=6 \bar{\delta}_{r}^{5}-15 \bar{\delta}_{r}^{4}+10 \bar{\delta}_{r}^{3}, \bar{\delta}_{r}=\frac{\delta_{r}}{\varepsilon r_{E}}, \delta_{r}=r_{E}-\frac{P}{W} \sin \left(L-\Omega_{c}\right) .
$$

Note that $\varepsilon r_{E}$ is the distance between the two closest shadow cone frontiers, $\delta_{r}$ is the distance between $x(t)$ and the imaginary shadow cone, and $\bar{\delta}_{r}$ is a normalized distance. 
According to the PMP, every minimizing trajectory $y^{\varepsilon}(\cdot)$ defined on $\left[0, t_{f}^{\varepsilon}\right]$, solution of the resulting $(\mathbf{O C P})_{\varepsilon}$, is the projection of an extremal $\left(y^{\varepsilon}(\cdot), p^{\varepsilon}(\cdot), p^{0 \varepsilon}, u^{\varepsilon}(\cdot)\right)$ solution of (1.10) and (1.11), with the same transversality conditions as (HOCP).

Note that the control domain $b(\varepsilon, \cdot, \cdot) \Omega$ of $(\mathbf{O C P})_{\varepsilon}$ contains the control domain of (HOCP). It follows that any feasible control strategy of (HOCP) is also feasible for $(\mathbf{O C P})_{\varepsilon}$, and therefore $t_{f}^{\varepsilon} \leqslant t_{f}$, for every $\varepsilon>0$.

3.4. Numerical simulations. We consider the following boundary conditions:

$$
\begin{aligned}
& x(0)=\left(P_{0}, e_{x 0}, e_{y 0}, L_{0}\right)=(11.625 \mathrm{Mm}, 0.75,0, \pi), m(0)=1500 \mathrm{~kg}, \\
& x\left(t_{f}\right)=\left(P_{f}, e_{x f}, e_{y f}, L_{f}\right)=(42.165 \mathrm{Mm}, 0,0, \text { free }), m\left(t_{f}\right) \text { free },
\end{aligned}
$$

corresponding to a low highly eccentric initial orbit and to the geostationary final orbit. The various numerical parameters are $\mu=5165.86248 \mathrm{Mm}^{3} / \mathrm{h}^{2}, \beta \approx$ $0.028325 \mathrm{~h} / \mathrm{Mm}, r_{E}=6.378 \mathrm{Mm}$, and $\Omega_{c 0}=0 \mathrm{rad}$. The value of the maximal thrust is chosen between 0.1 and 60 Newton in the next numerical simulations.

A single shooting method is used to solve the regularized and hybrid problems. The extremal flow is integrated using the adaptative step integrator DOPRI (see $[20])$. This integrator is particularly useful to take into account the jump conditions since its dense output allows us to accurately locate the crossing times. The Newton like method underlying our shooting method is the routine HYBRD of the minpack library.

We combine the shooting method with a continuation on the values of $T_{\max }$. The unconstrained problem is easily solved for a large value of $T_{\max }$ and the obtained solution is used to successfully initialize the solving of the regularized problem for a large value of $\varepsilon$, say $\varepsilon=0.9$. This solution enables us to solve the regularized problem for any desired (low) value of $T_{\max }$, by a decreasing continuation on the parameter $T_{\max }$. Then, another decreasing continuation on the regularization parameter $\varepsilon$ leads to the solution of the regularized problem with a small value of $\varepsilon$. If $\varepsilon>0$ is small enough then the latter solution falls into the domain of convergence of the shooting method associated with (HOCP) and is thus used to successfully initialize the shooting method on the hybrid problem. This illustrates the convergence properties derived in our main results.

We report on Table 3.1 the values of $t_{f}^{\varepsilon}$ for several values of $\varepsilon$ and of $T_{\max }$. As

\begin{tabular}{c|c|c|c|c}
$\varepsilon \backslash T_{\max }$ & $60 N$ & $10 N$ & $1 N$ & $0.1 N$ \\
\hline No cone & 14.281 & 80.782 & 806.831 & 7985.138 \\
\hline 0.9 & 14.337 & 81.307 & 810.273 & 8209.665 \\
\hline 0.5 & 14.359 & 81.521 & 812.607 & 8298.558 \\
\hline 0.1 & 14.383 & 81.750 & 815.143 & 8382.722 \\
\hline \hline 0 & 14.389 & 81.810 & 815.813 & 8406.773 \\
& \multicolumn{4}{|c}{ TABLE 3.1 }
\end{tabular}

expected, the minimal time $t_{f}^{\varepsilon}$ of $(\mathbf{O C P})_{\varepsilon}$ converges to $t_{f}$ as $\varepsilon$ tends to 0 . We report on Table 3.2 several numerical results illustrating the convergence of the state, adjoint vector, and control. It is important to notice on this table that it is not the magnitude of the differences but rather their decrease that illustrates the convergence. Graphic 


\begin{tabular}{c|c|c|c|c}
$\cdot \backslash \varepsilon$ & 0.9 & 0.5 & 0.1 & 0.05 \\
\hline$\left\|P^{\varepsilon}(\cdot)-P(\cdot)_{\mid\left[0, t_{f}^{\varepsilon}\right]}\right\|_{\infty}$ & 0.3799 & 0.1969 & 0.1529 & 0.0840 \\
\hline$\left\|e_{x}^{\varepsilon}(\cdot)-e_{x}(\cdot)_{\left[0, t_{f}^{\varepsilon}\right]}\right\|_{\infty}$ & 0.0270 & 0.0148 & 0.0034 & 0.0017 \\
\hline$\left\|e_{y}^{\varepsilon}(\cdot)-e_{y}(\cdot)_{\mid\left[0, t_{f}^{\varepsilon}\right]}\right\|_{\infty}$ & 0.0080 & 0.0047 & 0.0011 & 0.0005 \\
\hline$\left\|L^{\varepsilon}(\cdot)-L(\cdot)_{\mid\left[0, t_{f}^{\varepsilon}\right.}\right\|_{\infty}$ & 28.2347 & 28.5897 & 2.7437 & 1.2967 \\
\hline$\left\|m^{\varepsilon}(\cdot)-m(\cdot)_{\mid\left[0, t_{f}^{\varepsilon}\right]}\right\|_{\infty}$ & 7.8880 & 4.4118 & 0.9477 & 0.4971 \\
\hline \hline$\left|p_{P}^{\varepsilon}(0)-p_{P}(0)\right|$ & 95.3 & 78.6 & 8.19 & 4.97 \\
\hline$\left|p_{e_{x}}^{\varepsilon}(0)-p_{e_{x}}(0)\right|$ & 2886 & 2394 & 236.9 & 144.7 \\
\hline$\left|p_{e_{y}}^{\varepsilon}(0)-p_{e_{y}}(0)\right|$ & 59.3 & 40.4 & 6.84 & 3.55 \\
\hline$\left|p_{L}^{\varepsilon}(0)-p_{L}(0)\right|$ & 1 & 0.9 & 0.088 & 0.055 \\
\hline$\left|p_{m}^{\varepsilon}(0)-p_{m}(0)\right|$ & 0.6 & 0.5 & 0.05 & 0.029 \\
\hline \hline$\left\|u^{\varepsilon}(\cdot)-u(\cdot)_{\mid\left[0, t_{f}^{\varepsilon}\right]}\right\|_{L^{1}} / t_{f}^{\varepsilon}$ & 0.8857 & 0.9435 & 0.8762 & 0.4272
\end{tabular}

Component-wise $\|\cdot\|_{\infty}$-difference between $x^{\varepsilon}(\cdot)$ and $x(\cdot)$ (restricted to $\left.\left[0, t_{f}^{\varepsilon}\right]\right)$, difference between initial adjoint vectors, and averaged $L^{1}$-difference of controls for $T_{\max }=0.1 \mathrm{~N}$.

evidence of the convergence properties of $p^{\varepsilon}(\cdot)$ and $u^{\varepsilon}(\cdot)$ is illustrated on Figure 3.3 for $T_{\max }=60 \mathrm{~N}$. The reason for choosing such a large value of $T_{\max }$ is that the orbit transfer exhibits only one passage in the Earth's shadow cone, and the result is therefore more visible on the figure. Furthermore, we use the large value $\varepsilon=0.9$ so as to observe clearly the continuity of $p^{\varepsilon}(\cdot)$. On this figure, we observe that $p^{\varepsilon}(\cdot)$ already mimics the behavior of $p(\cdot)$. Moreover, the adjoint vector of the hybrid problem has the expected jump.
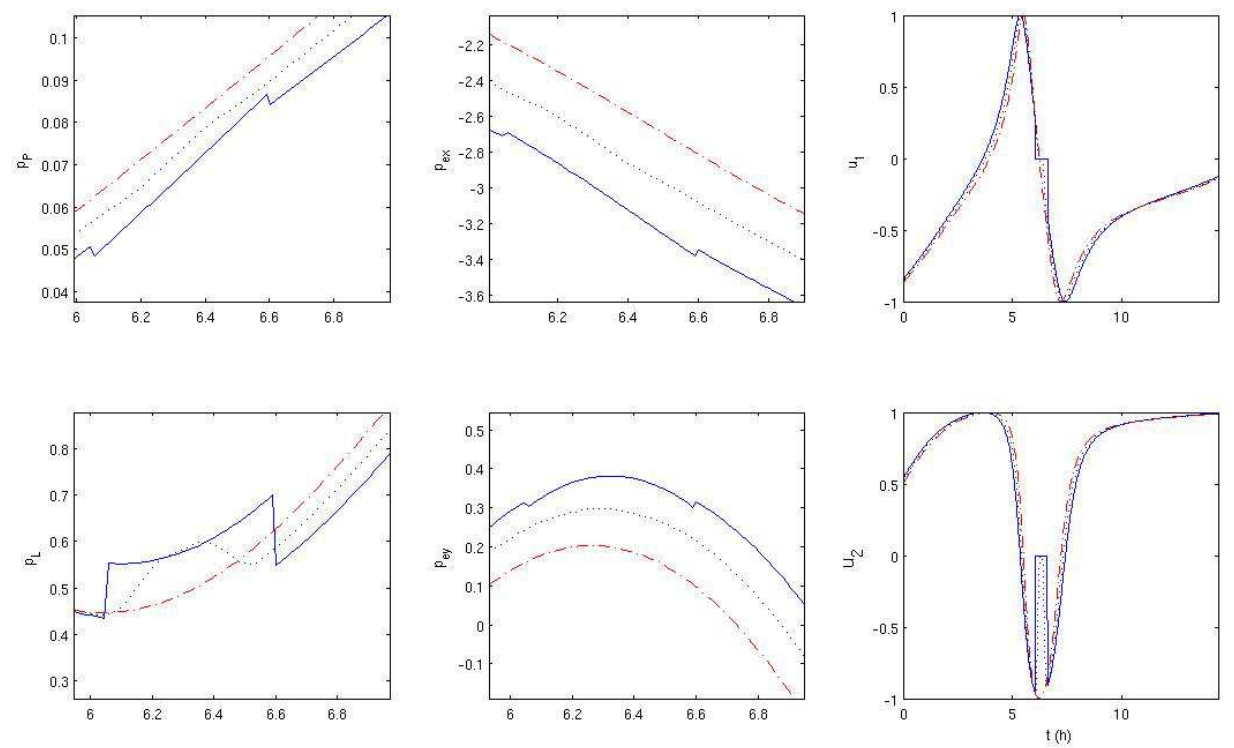

FIG. 3.3. Control and zoom on the adjoint vector $p_{x}(\cdot)$ for the unconstrained (dashed), regularized $\left(\varepsilon=0.9\right.$, dotted) and hydrid (plain) problem, $T_{\max }=60 \mathrm{~N}$. 
Finally, Figure 3.4 shows the evolution of the zero of the shooting function with respect to $\varepsilon$. The starred point represents the zero of the shooting function associated with (HOCP). The regularity of the zero path is another hint of the nice convergence properties.
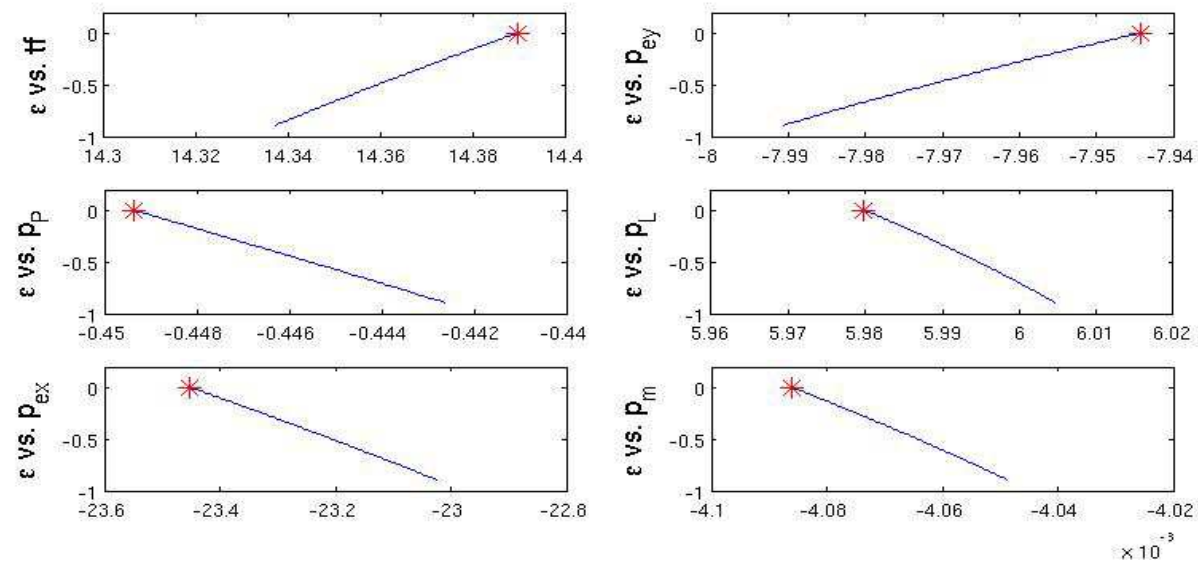

FIG. 3.4. Zero Path w.r.t. $\varepsilon$ for $T_{\max }=60 \mathrm{~N}$.

4. Appendix: conic implicit function theorem with parameters. Recall that a mapping $f: C \rightarrow F$, where $F$ is a Banach space and $C$ is a subset of a normed vector space $E$, is said strictly differentiable at some point $x_{0} \in C$ whenever there exists a linear continuous mapping from $E$ into $F$, denoted $d f\left(x_{0}\right)$ or $\frac{\partial f}{\partial x}\left(x_{0}\right)$ (and called differential of $f$ at $x_{0}$ ), such that

$$
f(y)-f(x)=d f\left(x_{0}\right) \cdot(y-x)+\|y-x\| \varepsilon(x, y),
$$

for all $x, y \in C$, where $\varepsilon(x, y)$ tends to 0 as $\left\|x-x_{0}\right\|+\left\|y-x_{0}\right\|$ tends to 0 .

Note that, if $f$ is $C^{1}$ then it is strictly differentiable at every point, for every subset $C$.

The notion of strict differentiability permits to derive a conic version of the implicit function theorem, useful in this paper. Hereafter we add moreover a dependence to some parameter.

Theorem 4.1. Consider a mapping

$$
\begin{aligned}
F: \mathbb{R}_{+} \times \mathbb{R}_{+}^{m} & \rightarrow \mathbb{R}^{n} \\
(\varepsilon, x) & \mapsto F(\varepsilon, x)
\end{aligned}
$$

satisfying the following assumptions:

- $F(0,0)=0$;

- $F$ is continuous;

- for every $\varepsilon \geqslant 0, F$ is strictly differentiable with respect to $x$ at 0 , and $\frac{\partial F}{\partial x}$ is continuous with respect to $\varepsilon$;

- $\frac{\partial F}{\partial x}(0,0) \cdot \mathbb{R}_{+}^{m}=\mathbb{R}^{n}$.

Then, there exist $\varepsilon_{0}>0$, a neighborhood $V$ of 0 in $\mathbb{R}^{n}$, and a function $g:\left[0, \varepsilon_{0}\right] \times V \rightarrow$ $\mathbb{R}^{m}$ with values in $\mathbb{R}_{+}^{m}$, such that

$$
F(\varepsilon, g(\varepsilon, y))=y
$$


for every $\varepsilon \in\left[0, \varepsilon_{0}\right]$ and every $y \in V$.

Proof. We start the proof with the following general fact.

Lemma 4.2. Let $\ell: \mathbb{R}^{m} \rightarrow \mathbb{R}^{n}$ be a linear mapping such that $\ell\left(\mathbb{R}_{+}^{m}\right)=\mathbb{R}^{n}$. Then,

(i) the intersection $(0,+\infty)^{m} \cap \operatorname{ker} \ell$ is nontrivial;

(ii) $m \geqslant n+1$;

(iii) there exists a $n$-dimensional subspace $W$ of $\mathbb{R}^{m}$ such that $\ell_{\mid W}: W \rightarrow \mathbb{R}^{n}$ is an isomorphism.

Proof. Let $\left(e_{1}, \ldots, e_{n}\right)$ be a basis of $\mathbb{R}^{n}$. Since $\ell\left(\mathbb{R}_{+}^{m}\right)=\mathbb{R}^{n}$, for every $i \in$ $\{1, \ldots, n\}$ there exists $v_{i} \in \mathbb{R}_{+}^{m}$ such that $e_{i}=\ell\left(v_{i}\right)$, and since $\ell$ is continuous, up to modifying slightly the vectors $e_{i}$ one can assume that $v_{i} \in(0,+\infty)^{m}$. Obviously, the vectors $v_{1}, \ldots, v_{n}$ are linearly independent. Set $e_{0}=-\sum_{i=1}^{n} e_{i}$. Since $\ell\left(\mathbb{R}_{+}^{m}\right)=\mathbb{R}^{n}$, there exists $v_{0} \in \mathbb{R}_{+}^{m}$ such that $e_{0}=\ell\left(v_{0}\right)$, and as previously one can assume that $v_{0} \in(0,+\infty)^{m}$. Note that, necessarily, $v_{0} \neq-\sum_{i=1}^{n} v_{i}$. Now, set $v=\frac{1}{n+1} \sum_{i=0}^{n} v_{i}$. Then, the vector $v$ is nontrivial, belongs to $(0,+\infty)^{m}$, and satisfies clearly $\ell(v)=0$. The point (i) follows. In particular, the kernel of $\ell$ is nontrivial, and this yields the point (ii). Consider the subspace of $\mathbb{R}^{m}$

$$
W=\operatorname{Span}\left\{v_{i}-v \mid i=0, \ldots, n\right\} .
$$

Clearly, $W$ is of dimension $n$, and, since $\ell\left(v_{i}-v\right)=e_{i}$ for every $i \in\{1, \ldots, n\}$, the point (iii) follows.

Applying this lemma to $\ell=\frac{\partial F}{\partial x}(0,0)$ yields the existence of a nontrivial vector $v \in(0,+\infty)^{m}$ such that $\ell(v)=0$, and the existence of a $n$-dimensional subspace $W$ of $\mathbb{R}^{m}$ such that $\ell_{\mid W}: W \rightarrow \mathbb{R}^{n}$ is an isomorphism.

For $\delta>0$, denote by $\bar{B}_{\mathbb{R}^{n}}(0, \delta)$ the closed ball of $\mathbb{R}^{m}$ centered at 0 and with radius $\delta$. Let $\delta>0$ small enough such that $v+B_{\delta} \subset(0,+\infty)^{m}$, where $B_{\delta}=W \cap \bar{B}_{\mathbb{R}^{n}}(0, \delta)$. The set $U_{\delta}=\ell\left(B_{\delta}\right)$ is then a closed neighborhood of 0 in $\mathbb{R}^{n}$.

For every $\varepsilon \geqslant 0$ and all $y, u \in \mathbb{R}^{n}$, set

$$
\Phi(\varepsilon, y, u)=u-F\left(\varepsilon, \ell_{\mid W}^{-1}(u)\right)+y .
$$

There holds $\Phi(0,0,0)=0$, and, for every $\varepsilon \geqslant 0$ and all $y, u_{1}, u_{2} \in \mathbb{R}^{n}$, one has

$$
\begin{aligned}
\Phi\left(\varepsilon, y, u_{1}\right)-\Phi\left(\varepsilon, y, u_{2}\right) & =u_{1}-u_{2}+F\left(\varepsilon, \ell_{\mid W}^{-1}\left(u_{2}\right)\right)-F\left(\varepsilon, \ell_{\mid W}^{-1}\left(u_{1}\right)\right) \\
& =u_{1}-u_{2}+\frac{\partial F}{\partial x}(\varepsilon, 0) \cdot \ell_{\mid W}^{-1}\left(u_{2}-u_{1}\right)+\left\|u_{2}-u_{1}\right\| \varepsilon\left(u_{1}, u_{2}\right),
\end{aligned}
$$

where $\varepsilon\left(u_{1}, u_{2}\right)$ tends to 0 as $\left\|u_{1}\right\|+\left\|u_{2}\right\|$ tends to 0 . Since $\frac{\partial F}{\partial x}$ is continuous with respect to $\varepsilon$, it follows that the mapping $\frac{\partial F}{\partial x}(\varepsilon, 0) \cdot \ell_{\mid W}^{-1}$ is close to the identity whenever $\varepsilon$ is small enough. Therefore, there exist $k \in(0,1)$ and $\varepsilon_{0}>0$ such that, for every $\varepsilon \in\left[0, \varepsilon_{0}\right]$, for every $y \in \mathbb{R}^{n}$, the mapping $u \mapsto \Phi(\varepsilon, y, u)$ is a $k$-Lipschitzian on $\mathbb{R}^{n}$ (since $k<1$ it is a contraction).

LEMma 4.3. If $\delta, \varepsilon$ and $\|y\|$ are small enough, then the mapping $u \mapsto \Phi(\varepsilon, y, u)$ maps $U_{\delta}$ into itself.

Proof. First of all, we claim that

$$
B_{\mathbb{R}^{n}}\left(0, \frac{\delta}{\left\|\ell_{\mid W}^{-1}\right\|}\right) \subset U_{\delta} .
$$


Here, $B_{\mathbb{R}^{n}}\left(0, \frac{\delta}{\left\|\ell_{\mid W}^{-1}\right\|}\right)$ is the open ball of $\mathbb{R}^{n}$ centered at 0 , with radius $\frac{\delta}{\left\|\ell_{\mid W}^{-1}\right\|}$, and $\left\|\ell_{\mid W}^{-1}\right\|$ is the norm of the linear continuous mapping $\ell_{\mid W}^{-1}$. Indeed, for every element $u$ of this ball, one has $u=\ell \circ \ell_{\mid W}^{-1}(u)$, and by construction, $\left\|\ell_{\mid W}^{-1}(u)\right\| \leqslant \delta$.

Secondly, proceeding as previously, there holds

$$
\begin{aligned}
u-F\left(\varepsilon, \ell_{\mid W}^{-1}(u)\right) & =u+F(\varepsilon, 0)-F\left(\varepsilon, \ell_{\mid W}^{-1}(u)\right)-F(\varepsilon, 0) \\
& =u-\frac{\partial F}{\partial x}(\varepsilon, 0) \cdot \ell_{\mid W}^{-1}(u)+\|u\| \varepsilon(u)-F(\varepsilon, 0),
\end{aligned}
$$

and hence there exists $\eta>0$ such that, for every $u \in \mathbb{R}^{n}$ satisfying $\|u\| \leqslant \eta$,

$$
\left\|u-F\left(\varepsilon, \ell_{\mid W}^{-1}(u)\right)\right\| \leqslant \frac{1}{3\|\ell\|\left\|\ell_{\mid W}^{-1}\right\|}\|u\|+\|F(\varepsilon, 0)\| .
$$

Now, choose $\delta$ small enough so that $\|\ell\| \leqslant \eta$. Then, for every $u \in U_{\delta}$, one has $\|u\| \leqslant\|\ell\| \delta \leqslant \eta$, and thus the inequality (4.2) holds for every $u \in U_{\delta}$.

Choose $y$ small enough so that $\|y\| \leqslant \frac{\delta}{3\left\|\ell_{\mid W}^{-1}\right\|}$. Then, for every $u \in U_{\delta}$, using (4.2),

$$
\begin{aligned}
\|\Phi(\varepsilon, y, u)\| & \leqslant\left\|u-F\left(\varepsilon, \ell_{\mid W}^{-1}(u)\right)\right\|+\|y\| \\
& \leqslant \frac{1}{3\|\ell\|\left\|\ell_{\mid W}^{-1}\right\|}\|\ell\| \delta \leqslant \eta+\|F(\varepsilon, 0)\|+\frac{\delta}{3\left\|\ell_{\mid W}^{-1}\right\|},
\end{aligned}
$$

and hence, since $F(0,0)=0$, if $\varepsilon$ is small enough so that $\|F(\varepsilon, 0)\| \leqslant \frac{\delta}{3\left\|\ell_{\mid W}^{-1}\right\|}$, then

$$
\|\Phi(\varepsilon, y, u)\| \leqslant \frac{\delta}{\left\|\ell_{\mid W}^{-1}\right\|} .
$$

Using (4.1), it follows that $\Phi(\varepsilon, y, u) \in U_{\delta}$. The lemma is proved.

Using Lemma 4.3, the theorem then follows from the application of the usual Banach fixed point theorem to the contraction mapping $u \mapsto \Phi(\varepsilon, y, u)$ with parameters $(\varepsilon, y)$. प

REMARK 4.4. We stress on that the notion of strict differentiability is crucial here to derive such a conic version of the implicit function theorem with parameters. It permits to derive a proof using the standard Banach fixed point theorem, which is well adapted to the case of parameters, contrarily to (e.g.) the Brouwer fixed point theorem that would not permit to derive a continuous section as in the statement of Theorem 4.1. In our article, getting such a continuous section is important in our proofs; topological arguments would not suffice.

\section{REFERENCES}

[1] A. Agrachev, Y. Sachkov, Control theory from the geometric viewpoint, Encyclopaedia of Mathematical Sciences 87, Control Theory and Optimization, II, Springer-Verlag, Berlin, 2004.

[2] M. Alamir, A benchmark for optimal control problem solvers for hybrid nonlinear systems, Automatica J. IFAC 42 (2006), no. 9, 1593-1598.

[3] J.-P. Aubin, H. Frankowska, Set-Valued Analysis, Birkhäuser, Boston, 1990.

[4] B. Bonnard, J.-B. Caillau, E. Trélat, Geometric optimal control of elliptic Keplerian orbits, Discrete Cont. Dyn. Syst. 5 (2005), no. 4, 929-956.

[5] B. Bonnard, L. Faubourg, E. Trélat, Mécanique céleste et contrôle des véhicules spatiaux, Springer, Collection "Mathématiques et Applications", 2006, 276 pages. 
[6] M. S. Branicky, V. S. Borkar, S. K. Mitter, A unified framework for hybrid control: model and optimal control theory, IEEE Trans. Autom. Control 43 (1998), no. 1, 31-45.

[7] A. Bressan, Y. Huong, Optimal control problems on stratified domains, Networks and Heterogeneous Media 2 (2007), no.2, 313-331.

[8] J.-B. Caillau, J. Noailles, Coplanar control of a satellite around the Earth, ESAIM Cont. Optim. Calc. Var. 6 (2001), 239-258.

[9] P. Cannarsa, C. Sinestrari, Semiconcave functions, Hamilton-Jacobi equations, and optimal control, Progress in Nonlinear Differential Equations and their Applications, 58, Birkhäuser Boston, Inc., Boston, MA, 2004.

[10] L. Cesari, Optimization - theory and applications. Problems with ordinary differential equations, Applications of Mathematics 17, New York, Springer-Verlag, 1983.

[11] F. H. Clarke, R. Vinter, The relationship between the maximum principle and dynamic programming, SIAM Journal on Control and Optimization, 25 (1987), no. 5, 1291-1311.

[12] C. D'Apice, M. Garavello, R. Manzo, B. Piccoli, Hybrid optimal control: case study of a car with gears, Internat. J. Control 76 (2003), no. 13, 1272-1284.

[13] A.V. Dmitruk, A.M. Kaganovich, The hybrid maximum principle is a consequence of Pontryagin maximum principle, Systems Control Lett. 57 (2008), no. 11, 964-970.

[14] A.L. Dontchev, Perturbations, Approximations and Sensitivity Analysis of Optimal Control Systems, Lecture Notes in Control and Information Sciences 52, Springer (1983).

[15] Ch. Ferrier, R. Epenoy, Optimal control for engines with electro-ionic propulsion under constraint of eclipse, Acta Astonautica 48, no. 4 (2001), 181-192.

[16] M. Garavello, B. Piccoli, Hybrid necessary principle, SIAM Journal on Control and Optimization 43 (2005), no. 5, 1867-1887.

[17] J. Gergaud, T. Haberkorn, Homotopy method for minimum consumption orbit transfer problem, ESAIM Cont. Optim. Calc. Var. 12 (2006), 294-310.

[18] J. Gergaud, T. Haberkorn, P. Martinon, Low thrust minimum fuel orbital transfer: an homotopic approach, J. Guidance Cont. Dyn. 27, 6 (2004), 1046-1060.

[19] W. Hager, Lipschitz continuity for constrained processes, SIAM Journal Cont. Optim. 17 (1979), no. 3, 321-338.

[20] E. Hairer, S.P. Norsett, G. Wanner, Solving Ordinary Differential Equations I. Nonstiff Problems. 2nd edition, Springer series in computational mathematics, Springer Verlag, 1993.

[21] J.-H. R. Kim, H. Maurer, Sensitivity analysis of optimal control problems with bang-bang controls, Proceedings of the 42nd IEEE Conference on Decision and Control, Maui, IEEE Control Society (2003), 3281-3286.

[22] E. B. Lee, L. Markus, Foundations of optimal control theory, John Wiley, New York, 1967.

[23] K. Malanowski, Differentiability with respect to parameters of solutions to convex programming problems, Mathematical Programming, Springer (1985) vol. 33, no. 3, 352-361.

[24] P. Martinon, J. Gergaud, Using switching detection and variational equations for the shooting method, Optimal Cont. Appl. Methods 28, no. 2 (2007), 95-116.

[25] H. Maurer, H. J. Pesch, Solution Differentiability for Parametric Nonlinear Control Problems with Control-State Constraints, SIAM Journal Cont. Optim. (1994), no. 32, 285-309.

[26] H. Maurer, G. Vossen, Sufficient conditions and sensitivity analysis for optimal bang-bang control problems with state constraints, Proceedings of the 23rd IFIP Conference on System Modeling and Optimization, Cracow, Poland, July 2007, Springer Verlag (2009), 82-99.

[27] L. Pontryagin, V. Boltyanskii, R. Gramkrelidze, E. Mischenko, The mathematical theory of optimal processes, Wiley Interscience, 1962.

[28] P. Riedinger, C. Iung, F. Kratz, An optimal control approach for hybrid systems, European Journal of Control, 9 (2003), no.5, 449-458.

[29] L. Rifford, E. Trélat, Morse-Sard type results in sub-Riemannian geometry, Math. Ann. 332 (2005), no. 1, 145-159.

[30] L. Rifford, E. Trélat, On the stabilization problem for nonholonomic distributions, J. Eur. Math. Soc., 11 (2009), no. 2, 223-255.

[31] S. Sager, Reformulations and algorithms for the optimization of switching decisions in nonlinear optimal control, Journal of Process Control 19 (2009), 1238-1247.

[32] M. S. Shaikh, P. E. Caines, On the hybrid optimal control problem: theory and algorithms, IEEE Trans. Automat. Control 52 (2007), no. 9, 1587-1603.

[33] C. J. Silva, E. Trélat, Smooth regularization of bang-bang optimal control problems, Preprint Hal (2009), 20 pages, to appear in IEEE Trans. Autom. Control.

[34] G. Stefani, Regularity properties of the minimum-time map, Nonlinear synthesis (Sopron, 1989), 270-282, Progr. Systems Control Theory, 9, Birkhäuser Boston, Boston, MA, 1991.

[35] J. Stoer, R. Bulirsch, Introduction to Numerical Analysis, Springer-Verlag, Berlin-HeidelbergNew York, 1983. 
[36] H.J. Sussmann, A nonsmooth hybrid maximum principle, Stability and stabilization of nonlinear systems (Ghent, 1999), 325-354, Lecture Notes in Control and Inform. Sci. 246, Springer, London, 1999.

[37] E. Trélat, Some properties of the value function and its level sets for affine control systems with quadratic cost, J. Dyn. Cont. Syst. 6 (2000), no. 4, 511-541.

[38] A. Visintin, Strong convergence results related to strict convexity, Comm. Partial Differential Equations 9 (1984), no. 5, 439-466.

[39] X. Xu, P. J. Antsaklis, Optimal control of switched systems via nonlinear optimization based on direct differentiation of value functions, International Journal of Control 75 (2002), no. $16,1406-1426$. 